\title{
Overhead and Spectral Efficiency of Pilot-Assisted Interference Alignment in Time-Selective Fading Channels
}

\author{
Ratheesh K. Mungara, Student Member, IEEE, Geordie George, Student Member, IEEE, \\ and Angel Lozano, Fellow, IEEE
}

\begin{abstract}
The spectral efficiency achievable by IA (interference alignment) in a $K$-user MIMO (multiple-input multipleoutput) interference channel is studied in the face of timeselective continuous fading explicitly estimated through pilotsymbol observations. The robustness of IA in such operationally relevant conditions is assessed through a joint optimization of the pilot overhead and the IA update interval, which are characterized-in high-power conditions-as solutions of a fixedpoint equation. Variations of the formulation are given for both FDD (frequency-division duplexing) and TDD (time-division duplexing), the former requiring explicit feedback of the fading estimates and the latter relying on fading reciprocity. For the FDD variation, analog feedback is considered. In addition to arbitrary numbers of users and antennas, and arbitrary temporal fading correlation functions, the derivations accommodate forward and reverse links with asymmetric power levels.
\end{abstract}

Index Terms-Interference alignment, time-selective fading, pilot-assisted transmission, spectral efficiency, pilot overhead, precoder update interval.

\section{INTRODUCTION}

With instantaneous and perfect CSI (channel-state information) at transmitters and receivers, IA (interference alignment) maximizes the number of interference-free signaling dimensions, i.e., the number of DoF (degrees of freedom) in a $K$ user interference channel [2]. Although recent results indicate that the instantaneous nature of the CSI may be dispensed with [3], the need for the CSI to be perfect seems unavoidable for the alignment to yield the corresponding number of DoF. This motivates the interest in assessing how IA might perform in the face of some inevitable CSI imperfections. Broadly speaking, the issue of imperfect CSI involves three intertwined aspects:

(1) Fundamentally, it cannot be decoupled from the reality of fading selectivity. If the fading were not selective, the CSI could be arbitrarily precise; it is the selectivity that curbs the number of observations of each fading realization making it impossible to gather perfect CSI.

(2) An arguably less fundamental, but, in practice, equally relevant matter is the utilization of pilot symbols to obtain the CSI at the receivers. Pilots imply overhead.

R. K. Mungara, G. George and A. Lozano are with the Department of Information and Communication Technologies, Universitat Pompeu Fabra (UPF), 08018 Barcelona, Spain. E-mail: \{ratheesh.mungara, geordie.george, angel.lozano\}@upf.edu. This work was supported by the European Project FET 265578 "HIATUS" and by the MINNECO Project TEC2012-34642. The contents of this paper were presented in part at the 2013 IEEE Global Communications Conference (GLOBECOM) [1].
(3) Finally, there is the issue of rendering the CSI gathered at the receivers available also to the transmitters; this is simpler with TDD (time-division duplexing), because of fading reciprocity, and more challenging with FDD (frequency-division duplexing), where explicit feedback is unavoidable.

Some studies on the performance of IA with imperfect CSI have been put forth recently. In reference to (1) above, [4] bounded, by both above and below, the sum spectral efficiency achievable by IA in the face of noisy CSI. The impact of imperfect CSI and spatial correlation were jointly quantified in [5], where an approximate closed-form expression for the corresponding SINR (signal-to-interference-plus-noise ratio) was derived. Also, [6] presented simulation results suggesting that IA may be very fragile when (1) is accounted for; however, these results were for unoptimized IA and disregarded pilot overhead.

In reference to (2), parameters such as the number of pilot symbols and their power were optimized to maximize a mutual information lower bound in [7], for the case of single-user MIMO (multiple-input multiple-output). Pilot-assisted transmission has subsequently been considered for IA, e.g., in [8], which studied the impact of pilot overhead in terms of DoF, and in [9], where it was argued that an unrestrained number of cooperating base stations can actually be detrimental because of excessive overhead.

Finally, in reference to (3), a variety of techniques have been proposed for CSI feedback in FDD systems [10]-[13]. The authors in [10] considered digital (quantized) feedback for single-antenna interference channels with Grassmannian codebooks utilized to quantize the channel coefficients. The results were subsequently extended to MIMO interference channels in [11]. It was shown in [10], [11] that the number of DoF can be preserved in the face of quantized feedback as long as the number of feedback bits scales with the SNR, a result that directly extends to interference channels the original findings of [14]. In [12], a novel CSI feedback scheme was proposed by exploiting the structure of the IA equations and the Grassmannian representation of the CSI; the authors showed that this scheme can achieve better spectral efficiency compared to a naïve approach (which simply quantizes the channel matrices independently) for a given number of feedback bits, but still requires that such number scales with the SNR in order to preserve the DoF. As an attractive alternative to digital feedback, analog feedback has been shown to sustain 
the number of DoF achieved by IA [13]. However, all the digital feedback analyses in [10]-[12] and the analog feedback analysis in [13] assumed perfect CSI at the receivers.

This paper jointly tackles (1)-(3) with the goal of studying the robustness of IA with pilot and IA configurations optimized as function of the fading selectivity, SNR, number of users, and other parameters of interest under both TDD and FDD. Additional related works include [15]-[17], which study (1)-(3) for the single-user and the broadcast channels. Particularly relevant is [18], which studies (1)-(3) for IA with block fading.

While, like [18], most of the aforementioned references invoke block-fading structures, once (1)-(3) are jointly considered and IA is to be optimized for minimum pilot and feedback overhead, it becomes important to resort to continuous (symbol-by-symbol) fading [19]-[21]. With this finer model of the fading selectivity in place, the steps that must be considered for a complete analysis of the IA operation are:

- Periodic transmission of forward pilots to allow for CSI gathering at the receivers. The pilot symbols made available for this task are referred to as common pilots.

- Computation of the receive filters on the basis of the foregoing CSI.

- CSI feedback, in the case of FDD, or exploitation of fading reciprocity, in the case of TDD with carefully calibrated transceivers, to convey the CSI to the transmitters. In the FDD case, analog feedback is our choice and the coherent detection of the feedback entails a further process of reverse channel estimation [18].

- Computation of the transmit precoders on the basis of the CSI acquired by the transmitters.

- Transmission through the precoders of additional pilots, termed dedicated pilots, in order to enable estimation of the precoded channel matrices at the receivers. ${ }^{1}$ This, in turn, enables coherent detection of the subsequent payload data.

- Finally, transmission of such payload data.

All of the above steps, and the ensuing overheads, are considered here, under continuous fading. The utilization of a continuous fading model releases the choice of the interval between IA updates, which in block fading is implicitly given by the size of the fading blocks whereas in our work it becomes a free parameter as it is in an actual system. We formulate the optimization of pilot and feedback overheads, and of the IA update interval, to maximize the effective sum spectral efficiency for arbitrary SNRs. This general formulation is followed by a simplified version thereof that is tailored to the high-SNR regime, where IA is mostly of interest. The impact of asymmetric SNRs in the forward and reverse links is also explored and, to gauge the extend to which the IA performance degrades because of the various overheads, pilotassisted TDMA is invoked as a baseline throughout the paper.

Several algorithms [22]-[24] are available for the design of IA precoders. Although closed-form expressions have been

\footnotetext{
${ }^{1}$ Alternatively, the dedicated pilots could be suppressed in favor of coded messages directly conveying the precoders to the receivers, but this approach would be somewhat more susceptible to fading selectivity and might incur larger overheads.
}

given for some specific cases [22], they are not available in general. Alternatively, iterative IA algorithms [23], [24] have been proposed and shown to converge. The iterative algorithms optimize the precoders and the receive filters to minimize the leakage interference by alternating between the forward and the reverse links. These algorithms can be either centralized or distributed.

- In centralized IA, all the base stations share their CSI. A central entity gathers the global CSI of all the forward and reverse links and computes the precoders. With global CSI available at that common point, iterations can be performed off-line at the central entity eliminating the need for over-the-air signaling.

- In distributed IA, there is no CSI sharing among the base stations and each transmitter locally computes its own precoder. The iterations must take place over the air, incurring further signaling overhead.

This paper considers the centralized version, which is the most favorable one for IA.

\section{System Model}

We consider the standard interference channel setting with $K$ transmitter-receiver pairs. Transmitters and receivers are equipped with $N_{\mathrm{t}}$ and $N_{\mathrm{r}}$ antennas, respectively, and each transmitter-receiver link conveys $d \leq \min \left(N_{\mathrm{t}}, N_{\mathrm{r}}\right)$ signal streams. The observed vector $\mathbf{y}_{k} \in \mathbb{C}^{N_{\mathrm{r}} \times 1}$ at receiver $k$ is

$$
\mathbf{y}_{k}=\sum_{j=1}^{K} \sqrt{\frac{P}{d}} \mathbf{H}_{k, j} \mathbf{x}_{j}+\mathbf{z}_{k}
$$

where $\mathbf{x}_{j} \in \mathbb{C}^{N_{\mathrm{t}} \times 1}$ is the signal from transmitter $j$ while $\mathbf{H}_{k, j} \in \mathbb{C}^{N_{\mathrm{r}} \times N_{\mathrm{t}}}$ is the channel matrix from transmitter $j$ to receiver $k$ and $P$ is the transmit power. The noise at receiver $k$ is $\mathbf{z}_{k} \sim \mathcal{N}_{\mathbb{C}}\left(\mathbf{0}, N_{0} \mathbf{I}_{N_{\mathrm{r}}}\right)$, where $\mathcal{N}_{\mathbb{C}}(\mathbf{m}, \mathbf{R})$ denotes a complex Gaussian random vector with mean $\mathbf{m}$ and covariance R. The entries of $\mathbf{H}_{k, j}$ are IID (independent identically distributed), drawn from $\mathcal{N}_{\mathbb{C}}(0,1)$. The signal at transmitter $k$ is $\mathbf{x}_{k}=\mathbf{V}_{k} \mathbf{b}_{k}$ where $\mathbf{V}_{k}=\left[\mathbf{v}_{k, 1}, \ldots, \mathbf{v}_{k, d}\right] \in \mathbb{C}^{N_{\mathrm{t}} \times d}$ is the unitary precoding matrix at the $k$ th transmitter and $\mathbf{b}_{k}=\left[b_{k, 1}, b_{k, 2}, \ldots, b_{k, d}\right]^{T} \in \mathbb{C}^{d \times 1}$ is the complex data symbol vector satisfying $\mathbb{E}\left[\mathbf{b}_{k} \mathbf{b}_{k}^{H}\right]=\mathbf{I}_{d}$. While the data symbols could be chosen from any distribution, we focus on Gaussian codebooks, which maximize the mutual information between $\mathbf{x}_{k}$ and $\mathbf{y}_{k}$ with perfect IA. Power is uniformly allocated across streams, which is asymptotically optimal at high SNR. At receiver $k$, the vector $\mathbf{y}_{k}$ is filtered by a unitary matrix $\mathbf{W}_{k}=\left[\mathbf{w}_{k, 1}, \ldots, \mathbf{w}_{k, d}\right] \in \mathbb{C}^{N_{\mathrm{r}} \times d}$.

The SINR of the $\ell$ th signal stream at receiver $k$ is given by

$$
\gamma_{k, \ell}=\frac{\frac{P}{d}\left|\mathbf{w}_{k, \ell}^{H} \mathbf{H}_{k, k} \mathbf{v}_{k, \ell}\right|^{2}}{\mathcal{I}_{k, \ell}+N_{0}\left\|\mathbf{w}_{k, \ell}\right\|_{2}^{2}}
$$

where

$$
\mathcal{I}_{k, \ell}=\sum_{\substack{m=1, m \neq \ell}}^{d} \frac{P}{d}\left|\mathbf{w}_{k, \ell}^{H} \mathbf{H}_{k, k} \mathbf{v}_{k, m}\right|^{2}+\sum_{\substack{j=1, m=1 \\ j \neq k}}^{K} \sum_{m=1}^{d} \frac{P}{d}\left|\mathbf{w}_{k, \ell}^{H} \mathbf{H}_{k, j} \mathbf{v}_{j, m}\right|^{2}
$$




\section{IntERFERENCE ALIGNMENT}

IA with $d$ DoF per user is achieved if and only if the precoding and receiving filters of user $k$ simultaneously satisfy

$$
\begin{array}{rlrl}
\mathbf{W}_{k}^{H} \mathbf{H}_{k, j} \mathbf{V}_{j}=\mathbf{0} & & \forall j \neq k \\
\operatorname{rank}\left(\mathbf{W}_{k}^{H} \mathbf{H}_{k, k} \mathbf{V}_{k}\right) & =d & & \forall k
\end{array}
$$

where (4) are zero-forcing conditions on the interference while (5) guarantees the required dimensionality for the desired signal space. For a given $d$, a different number of antennas is required for IA to be feasible with each $K$. If $\min \left(N_{\mathrm{t}}, N_{\mathrm{r}}\right) \geq 2 d$, then a necessary and sufficient condition for IA feasibility is [25]

$$
N_{\mathrm{t}}+N_{\mathrm{r}} \geq(K+1) d .
$$

\section{A. Perfect CSI}

With perfect CSI provided as side information, IA yields a $d$-dimensional interference-free channel for every intended link. Then, the SINR of the $\ell$ th stream at receiver $k$ is

$$
\gamma_{k, \ell}=\rho\left|\mathbf{w}_{k, \ell}^{H} \mathbf{H}_{k, k} \mathbf{v}_{k, \ell}\right|^{2}
$$

where $\rho=\frac{P}{d N_{0}}$ is the average per-stream SNR. Since $\mathbf{V}_{k}$ and $\mathbf{W}_{k}$ are unitary and independent of $\mathbf{H}_{k, k}$, the effective channels $\mathbf{w}_{k, \ell}^{H} \mathbf{H}_{k, k} \mathbf{v}_{k, \ell}$ are complex Gaussian with unit variance. The average sum spectral efficiency is then

$$
\begin{aligned}
\bar{C}_{\text {sum }}(\rho) & =\sum_{k=1}^{K} \sum_{\ell=1}^{d} \mathbb{E}\left[\log _{2}\left(1+\rho\left|\mathbf{w}_{k, \ell}^{H} \mathbf{H}_{k, k} \mathbf{v}_{k, \ell}\right|^{2}\right)\right] \\
& =K d e^{1 / \rho} E_{1}\left(\frac{1}{\rho}\right) \log _{2} e
\end{aligned}
$$

where $E_{1}(\zeta)=\int_{1}^{\infty} t^{-1} e^{-\zeta t} d t$ is an exponential integral.

\section{B. Imperfect CSI}

With precoders and receive filters computed on the basis of estimated rather than actual fading coefficients, the signals become misaligned and interference leaks through. Figure 1 depicts the scheme considered in this paper for CSI acquisition and transfer.

Let us denote by $\hat{\mathbf{H}}_{k, j}$ an imperfect estimate of $\mathbf{H}_{k, j}$ at transmitter $k$ and by $\tilde{\mathbf{H}}_{k, j}=\mathbf{H}_{k, j}-\hat{\mathbf{H}}_{k, j}$ its estimation error. The IA precoders and receivers obtained on the basis of channel estimates are $\hat{\mathbf{V}}_{k}$ and $\hat{\mathbf{W}}_{k}$, for $k=1, \ldots, K$. At receiver $k$, the $\ell$ th signal stream is extracted by $\hat{\mathbf{w}}_{k, \ell}$ yielding

$$
\begin{aligned}
\hat{\mathbf{w}}_{k, \ell}^{H} \mathbf{y}_{k}= & \sum_{j=1}^{K} \sqrt{\frac{P}{d}} \hat{\mathbf{w}}_{k, \ell}^{H} \mathbf{H}_{k, j} \hat{\mathbf{v}}_{j} \mathbf{b}_{j}+\hat{\mathbf{w}}_{k, \ell}^{H} \mathbf{z}_{k} \\
= & \sum_{j=1}^{K} \sqrt{\frac{P}{d}} \hat{\mathbf{w}}_{k, \ell}^{H}\left(\hat{\mathbf{H}}_{k, j}+\tilde{\mathbf{H}}_{k, j}\right) \hat{\mathbf{V}}_{j} \mathbf{b}_{j}+\hat{\mathbf{w}}_{k, \ell}^{H} \mathbf{z}_{k} \\
= & \sqrt{\frac{P}{d}} \hat{\mathbf{w}}_{k, \ell}^{H} \hat{\mathbf{H}}_{k, k} \hat{\mathbf{v}}_{k, \ell} b_{k, \ell} \\
& +\sum_{j=1}^{K} \sum_{m=1}^{d} \sqrt{\frac{P}{d}} \hat{\mathbf{w}}_{k, \ell}^{H} \tilde{\mathbf{H}}_{k, j} \hat{\mathbf{v}}_{j, m} b_{j, m}+\hat{\mathbf{w}}_{k, \ell}^{H} \mathbf{z}_{k}
\end{aligned}
$$

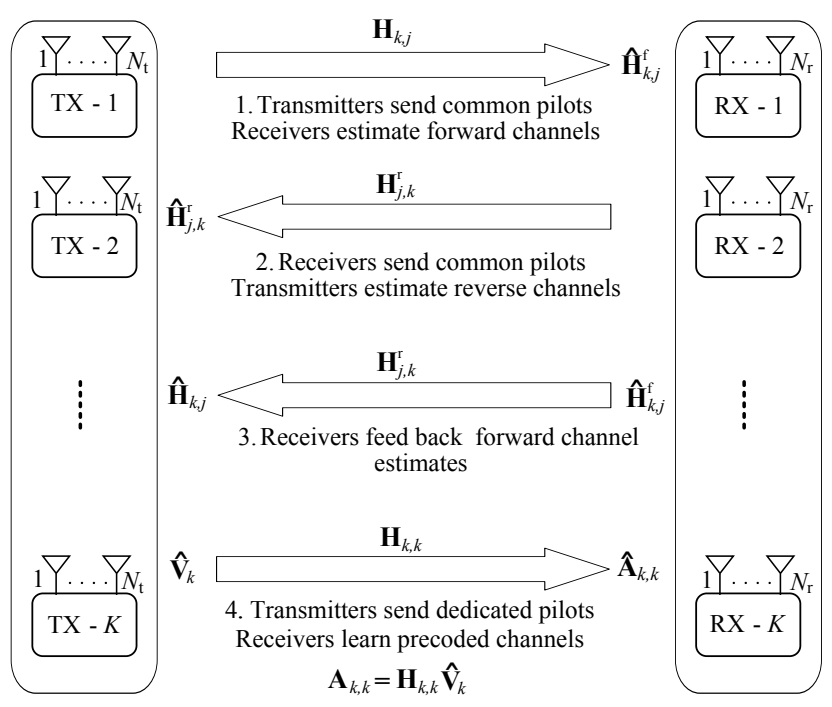

Fig. 1. CSI transfer with FDD in a $K$-user MIMO interference channel. Notation: $\hat{\mathbf{H}}_{k, j}^{\mathrm{f}}$ represents the estimate of forward channel $\mathbf{H}_{k, j}$ at receiver $k$; $\mathbf{H}_{j, k}^{\mathrm{r}}$ represents the reverse channel from receiver $k$ to transmitter $j$ and $\hat{\mathbf{H}}_{j, k}^{\mathrm{r}}$ is its estimate at transmitter $j$; $\hat{\mathbf{H}}_{k, j}$ denotes the estimate of $\mathbf{H}_{k, j}$ available at transmitter $k ; \hat{\mathbf{V}}_{j}$ is the IA precoder at transmitter $j ; \mathbf{A}_{k, k}$ denotes the precoded channel matrix from transmitter $k$ to receiver $k$ and $\hat{\mathbf{A}}_{k, k}$ is its estimate at receiver $k$.

where (11) follows from the fact that the IA solution computed on the basis of channel estimates satisfies $\hat{\mathbf{w}}_{k, \ell}^{H} \hat{\mathbf{H}}_{k, j} \hat{\mathbf{v}}_{j, m}=$ $0, \forall(k, \ell) \neq(j, m)$. If the decoders regard the channel estimates as the actual channel coefficients, as is customary, then the estimation error terms in (11) simply play the role of additional Gaussian noise [26]. In turn, $\hat{\mathbf{V}}_{k}$ and $\hat{\mathbf{W}}_{k}$ are unitary and independent of $\hat{\mathbf{H}}_{k, k}$ and $\tilde{\mathbf{H}}_{k, j}, \forall k, j$. However, $\hat{\mathbf{w}}_{k, \ell}^{H} \hat{\mathbf{H}}_{k, k} \hat{\mathbf{v}}_{k, \ell} b_{k, \ell}$ and $\hat{\mathbf{w}}_{k, \ell}^{H} \tilde{\mathbf{H}}_{k, j} \hat{\mathbf{v}}_{j, m} b_{j, m}$ are in general dependent when $(k, \ell)=(j, m)$. By invoking MMSE (minimum mean-square error) estimation, $\hat{\mathbf{H}}_{k, j}$ and $\tilde{\mathbf{H}}_{k, j}$ become uncorrelated and, given their Gaussian nature, mutually independent; this renders the aforementioned terms also independent for $(k, \ell)=(j, m)$. The entries of $\tilde{\mathbf{H}}_{k, j}$ are $\operatorname{IID} \mathcal{N}_{\mathbb{C}}\left(0, \sigma_{\tilde{\mathbf{H}}}^{2}\right)$ whereas the entries of $\hat{\mathbf{H}}_{k, j}$ are $\mathcal{N}_{\mathbb{C}}\left(0,1-\sigma_{\tilde{\mathbf{H}}}^{2}\right)$.

Then, the average sum spectral efficiency is ${ }^{2}$

$$
\begin{aligned}
\bar{C}_{\text {sum }}= & \sum_{k=1}^{K} \sum_{\ell=1}^{d} \mathbb{E}\left[\log _{2}(1+\right. \\
& \left.\left.\frac{\frac{P}{d}\left|\hat{\mathbf{w}}_{k, \ell}^{H} \hat{\mathbf{H}}_{k, k} \hat{\mathbf{v}}_{k, \ell}\right|^{2}}{N_{0}+\sum_{j=1}^{K} \sum_{m=1}^{d} \mathbb{E}\left[\frac{P}{d}\left|\hat{\mathbf{w}}_{k, \ell}^{H} \tilde{\mathbf{H}}_{k, j} \hat{\mathbf{v}}_{j, m}\right|^{2}\right]}\right)\right]
\end{aligned}
$$

with the outer expectation taken over the direct fading channels, $\hat{\mathbf{H}}_{k, k}$. We note that this is the spectral efficiency achieved with separate decoding of each same-link stream; for $d>1$, joint decoding of all same-link streams would be required for

\footnotetext{
${ }^{2}$ The spectral efficiency in (12) corresponds to decoders that, as mentioned earlier, regard the channel estimates as the actual channel coefficients, in which case the nearest-neighbor decoding rule applies. For decoders exploiting the joint distribution of the channel estimates and the corresponding estimation errors, (12) represents a lower bound to the achievable spectral efficiency [27]. The difference between these two quantities tends to be minute [28].
} 
optimality [4]. For $d=1$, as in all the examples in this paper, this becomes immaterial.

The precoder $\hat{\mathbf{V}}_{k}$ and the receive filter $\hat{\mathbf{W}}_{k}$ are designed such that $\hat{\mathbf{W}}_{k}^{H} \hat{\mathbf{H}}_{k, j} \hat{\mathbf{V}}_{j}=0$ for $j \neq k$ based on the channel estimates $\hat{\mathbf{H}}_{k, j}$ available at transmitter $k$. The mismatch error variance

$$
\mathbb{E}\left[\left|\hat{\mathbf{w}}_{k, \ell}^{H} \tilde{\mathbf{H}}_{k, j} \hat{\mathbf{v}}_{j, m}\right|^{2}\right]
$$

for $j \neq k$ is proportional to the channel estimation error variance, $\sigma_{\tilde{\mathbf{H}}}^{2}$. For its part, the desired signal estimation error variance

$$
\mathbb{E}\left[\left|\hat{\mathbf{w}}_{k, \ell}^{H} \tilde{\mathbf{H}}_{k, k} \hat{\mathbf{v}}_{k, \ell}\right|^{2}\right]
$$

depends only on the dedicated pilots, i.e., it is proportional to the precoded channel estimation error variance denoted by $\sigma_{\tilde{\mathrm{A}}}^{2}$ (cf. Fig. 1). Defining the effective average per-stream SINR as

$$
\rho_{\text {eff }}=\frac{\rho\left(1-\sigma_{\tilde{\mathbf{A}}}^{2}\right)}{1+\rho d\left((K-1) \sigma_{\tilde{\mathbf{H}}}^{2}+\sigma_{\tilde{\mathbf{A}}}^{2}\right)}
$$

the expression in (12) can be more compactly rewritten as

$$
\bar{C}_{\text {sum }}\left(\rho_{\text {eff }}\right)=\sum_{k=1}^{K} \sum_{\ell=1}^{d} \mathbb{E}\left[\log _{2}\left(1+\rho_{\text {eff }}\left|\mathbf{h}_{\text {eff }, \ell}\right|^{2}\right)\right]
$$

where $\mathbf{h}_{\text {eff }, \ell}=\hat{\mathbf{w}}_{k, \ell}^{H} \hat{\mathbf{H}}_{k, k} \hat{\mathbf{v}}_{k, \ell}$ is complex Gaussian and thus

$$
\bar{C}_{\text {sum }}\left(\rho_{\text {eff }}\right)=K d \log _{2}(e) e^{1 / \rho_{\text {eff }}} E_{1}\left(\frac{1}{\rho_{\text {eff }}}\right) .
$$

\section{Time Selectivity}

Next, to tie the analysis in the previous section with the dynamics of the fading, consider a discrete-time stationary fading process $h[n]$ governed by a given Doppler spectrum or, equivalently, by the corresponding time-domain correlation function $\epsilon[m]=\mathbb{E}\left[h[n] h^{*}[n+m]\right]$. This model applies to every entry of each of the channel matrices.

Fading selectivity implies that the IA solution computed at a given instant suffers growing misalignment as time evolves. Precisely, if the IA solution is computed at time 0 on the basis of current channel estimates, at time $n$ the signal extracted for stream $\ell$ at receiver $k$ will be

$$
\begin{aligned}
\hat{\mathbf{w}}_{k, \ell}^{H} \mathbf{y}_{k}[n]= & \sum_{j=1}^{K} \sqrt{\frac{P}{d}} \hat{\mathbf{w}}_{k, \ell}^{H}\left(\epsilon[n] \mathbf{H}_{k, j}[0]\right. \\
& \left.+\sqrt{1-\epsilon[n]^{2}} \mathbf{\Psi}_{k, j}\right) \hat{\mathbf{V}}_{j} \mathbf{b}_{j}+\hat{\mathbf{w}}_{k, \ell}^{H} \mathbf{z}_{k}
\end{aligned}
$$

where $\mathbf{H}_{k, j}[0]$ is the channel at time 0 and $\boldsymbol{\Psi}_{k, j}$, whose entries are $\mathcal{N}_{\mathbb{C}}(0,1)$ and independent of $\mathbf{H}_{k, j}[0]$, is the innovation between times 0 and $n$. Except where essential, time indices are dropped henceforth for the sake of notational compactness. Leveraging the derivations in Section III-B, (16) can be

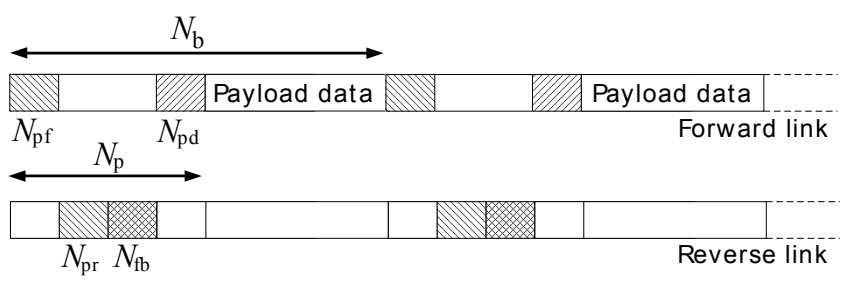

Fig. 2. Pilot overhead model.

rewritten as

$$
\begin{aligned}
\hat{\mathbf{w}}_{k, \ell}^{H} \mathbf{y}_{k}= & \sum_{j=1}^{K} \sqrt{\frac{P}{d}} \hat{\mathbf{w}}_{k, \ell}^{H}\left(\epsilon[n]\left(\hat{\mathbf{H}}_{k, j}+\tilde{\mathbf{H}}_{k, j}\right)\right. \\
& \left.+\sqrt{1-\epsilon[n]^{2}} \mathbf{\Psi}_{k, j}\right) \hat{\mathbf{V}}_{j} \mathbf{b}_{j}+\hat{\mathbf{w}}_{k, \ell}^{H} \mathbf{z}_{k} \\
= & \epsilon[n] \sqrt{\frac{P}{d}} \hat{\mathbf{w}}_{k, \ell}^{H} \hat{\mathbf{H}}_{k, k} \hat{\mathbf{v}}_{k, \ell} b_{k, \ell} \\
& +\sum_{j=1}^{K} \sum_{m=1}^{d} \sqrt{\frac{P}{d}} \hat{\mathbf{w}}_{k, \ell}^{H}\left(\epsilon[n] \tilde{\mathbf{H}}_{k, j}\right. \\
& \left.+\sqrt{1-\epsilon[n]^{2}} \mathbf{\Psi}_{k, j}\right) \hat{\mathbf{v}}_{j, m} b_{k, m}+\hat{\mathbf{w}}_{k, \ell}^{H} \mathbf{z}_{k}
\end{aligned}
$$

where (18) follows from the fact that $\hat{\mathbf{w}}_{k, \ell}^{H} \hat{\mathbf{H}}_{k, j} \hat{\mathbf{v}}_{j, m}=$ $0, \forall(k, \ell) \neq(j, m)$. In turn, the average sum mutual information at time $n$ is given by (19) in the next page with outer expectation over the direct fading channels. ${ }^{3}$ The effective average per-stream SINR at time $n$ is

$$
\rho_{\text {eff }}[n]=\frac{\rho \epsilon[n]^{2}\left(1-\sigma_{\tilde{\mathbf{A}}}^{2}\right)}{1+\rho d\left(K+\epsilon[n]^{2}\left((K-1) \sigma_{\tilde{\mathbf{H}}}^{2}-K+\sigma_{\tilde{\mathbf{A}}}^{2}\right)\right)}
$$

which follows from the uncorrelatedness of $\boldsymbol{\Psi}_{k, j}$ and $\tilde{\mathbf{H}}_{k, j}$. Thus, the average sum mutual information in (19) can be rewritten as

$$
\begin{aligned}
& \bar{I}_{\text {sum }}[n]=\sum_{k=1}^{K} \sum_{\ell=1}^{d} \mathbb{E}\left[\log _{2}\left(1+\rho_{\text {eff }}[n]\left|\mathbf{h}_{\text {eff }, \ell}\right|^{2}\right)\right] \\
& =K d \log _{2}(e) e^{1 / \rho_{\text {eff }}[n]} E_{1}\left(\frac{1}{\rho_{\text {eff }}[n]}\right) .
\end{aligned}
$$

Note that $\rho_{\text {eff }}[n]$ and $\bar{I}_{\text {sum }}[n]$ are decreasing functions of $n$, as one would expect. Furthermore, by setting $\epsilon[n]=1$ in (20) and (22), we recover (13) and (15) as derived for nonselective fading.

\section{Pilot Overhead Model}

\section{A. FDD}

With FDD, the forward and reverse links occupy distinct frequency bands separated by far more than the fading coherence bandwidth. Thus, the forward and reverse links are statistically independent and explicit CSI feedback is required.

\footnotetext{
${ }^{3}$ We resist referring to (19) as a spectral efficiency because it is only the average sum mutual information at a given lag $n$ relative to the time of computation of the IA solution. It is only once codewords are allowed to span many symbols at every lag that the operational significance of the average sum spectral efficiency is acquired, and this is deferred to subsequent sections.
} 


$$
\bar{I}_{\text {sum }}=\sum_{k=1}^{K} \sum_{\ell=1}^{d} \mathbb{E}\left[\log _{2}\left(1+\frac{\epsilon[n]^{2} \frac{P}{d}\left|\hat{\mathbf{w}}_{k, \ell}^{H} \hat{\mathbf{H}}_{k, k} \hat{\mathbf{v}}_{k, \ell}\right|^{2}}{N_{0}+\sum_{j=1}^{K} \sum_{m=1}^{d} \mathbb{E}\left[\frac{P}{d}\left|\hat{\mathbf{w}}_{k, \ell}^{H}\left(\epsilon[n] \tilde{\mathbf{H}}_{k, j}+\sqrt{1-\epsilon[n]^{2}} \mathbf{\Psi}_{k, j}\right) \hat{\mathbf{v}}_{j, m}\right|^{2}\right]}\right)\right]
$$

Figure 2 shows the pilot overhead model adopted in this paper. The IA solution is recomputed every $N_{\mathrm{b}}$ symbols, of which $N_{\mathrm{pf}}, N_{\mathrm{pr}}, N_{\mathrm{fb}}$ and $N_{\mathrm{pd}}$ are respectively reserved for forward common pilots, reverse common pilots, CSI feedback, and forward dedicated pilots; the rest of the symbols are available for payload data. The total overhead is hence $N_{\mathrm{p}}=N_{\mathrm{pf}}+N_{\mathrm{pr}}+N_{\mathrm{fb}}+N_{\mathrm{pd}}$. We make the very mild assumption that the fading remains constant over the pilot intervals ${ }^{4}$ and consider equal power allocation for pilot and data symbols.

1) Forward Common Pilots: Each transmitter $k$ broadcasts an orthogonal pilot sequence matrix $\boldsymbol{\Phi}_{k}$, such that $\boldsymbol{\Phi}_{k} \boldsymbol{\Phi}_{j}^{H}=$ $\delta_{k, j} \mathbf{I}_{N_{\mathrm{t}}}$, spanning $N_{\mathrm{pf}} \geq K N_{\mathrm{t}}$ symbols [29]. Each receiver estimates the channel matrices corresponding to each of the $K$ transmitters. The observation at receiver $k$ is

$$
\mathbf{Y}_{k}=\sqrt{\frac{N_{\mathrm{pf}} P}{N_{\mathrm{t}}}} \sum_{j=1}^{K} \mathbf{H}_{k, j} \mathbf{\Phi}_{j}+\mathbf{Z}_{k}
$$

where $\mathbf{Z}_{k} \in \mathbb{C}^{N_{\mathrm{r}} \times N_{\mathrm{pf}}}$ is a matrix of noise terms. We indicate the MMSE estimate of the forward channel $\mathbf{H}_{k, j}$ by $\hat{\mathbf{H}}_{k, j}^{\mathrm{f}}$ while denoting the corresponding estimation error matrix by $\tilde{\mathbf{H}}_{k, j}^{\mathrm{f}}$. Given the observation $\mathbf{Y}_{k}$,

$$
\hat{\mathbf{H}}_{k, j}^{\mathrm{f}}=\frac{\sqrt{\frac{N_{\mathrm{p} P} P}{N_{\mathrm{t}}}}}{N_{0}+\frac{N_{\mathrm{pf}} P}{N_{\mathrm{t}}}} \mathbf{Y}_{k} \boldsymbol{\Phi}_{j}^{H} \quad \forall j
$$

with entries of $\hat{\mathbf{H}}_{k, j}^{\mathrm{f}}$ are

$$
\mathcal{N}_{\mathbb{C}}\left(0, \frac{N_{\mathrm{pf}} P / N_{\mathrm{t}}}{N_{0}+N_{\mathrm{pf}} P / N_{\mathrm{t}}}\right)
$$

while the entries of $\tilde{\mathbf{H}}_{k, j}^{\mathrm{f}}$ are IID and

$$
\mathcal{N}_{\mathbb{C}}\left(0, \frac{N_{0}}{N_{0}+N_{\mathrm{pf}} P / N_{\mathrm{t}}}\right) .
$$

2) Reverse Common Pilots: Here, the roles of transmitters and receivers are interchanged and the receivers transmit orthogonal pilot sequences over $N_{\mathrm{pr}} \geq K N_{\mathrm{r}}$ symbols. Denote the reverse channel matrix between transmitter $k$ and receiver $j$ as $\mathbf{H}_{k, j}^{\mathrm{r}}$. The corresponding channel estimate is $\hat{\mathbf{H}}_{k, j}^{\mathrm{r}}$ with entries that distributed according to

$$
\mathcal{N}_{\mathbb{C}}\left(0, \frac{N_{\mathrm{pr}} P_{\mathrm{r}} / N_{\mathrm{r}}}{N_{\mathrm{o}}+N_{\mathrm{pr}} P_{\mathrm{r}} / N_{\mathrm{r}}}\right)
$$

while the error matrix is $\tilde{\mathbf{H}}_{k, j}^{\mathrm{r}}$ with entries conforming to

$$
\mathcal{N}_{\mathbb{C}}\left(0, \frac{N_{0}}{N_{0}+N_{\mathrm{pr}} P_{\mathrm{r}} / N_{\mathrm{r}}}\right)
$$

where $P_{\mathrm{r}}$ is the reverse link power. The ratio of reverse and forward link powers is defined as $\beta=P_{\mathrm{r}} / P$.

\footnotetext{
${ }^{4}$ The mildness of this assumption was validated by bounding the gap between the results with and without fading fluctuations within the pilot intervals; this gap was found to be very small, at most of a few percentage points (favoring IA) in terms of average spectral efficiency.
}

3) Analog CSI feedback: The forward channel estimates are transmitted via the reverse link by each receiver using unquantized quadrature-amplitude modulation over $N_{\mathrm{fb}}$ symbols. Each receiver $k$ sends its $N_{\mathrm{r}} \times K N_{\mathrm{t}}$ concatenated channel feedback matrix $\left[\hat{\mathbf{H}}_{k, 1}^{\mathrm{f}}, \ldots, \hat{\mathbf{H}}_{k, K}^{\mathrm{f}}\right]$ by post-multiplying it by a $K N_{\mathrm{t}} \times N_{\mathrm{fb}}$ unitary spreading matrix $\boldsymbol{\Theta}_{k}$ such that $\boldsymbol{\Theta}_{k} \boldsymbol{\Theta}_{j}^{H}=$ $\delta_{k, j} \mathbf{I}_{K N_{\mathrm{t}}}$. The spreading matrices from different receivers must be orthogonal to enable interference-free estimation, which imposes the constraint $N_{\mathrm{fb}} \geq K^{2} N_{\mathrm{t}}$. From receiver $k$, the transmitted channel feedback matrix can be written as [13], [30]

$$
\mathbf{X}_{k}=\sqrt{\frac{N_{\mathrm{fb}} P_{\mathrm{r}}}{K N_{\mathrm{t}} N_{\mathrm{r}}}\left(\frac{N_{\mathrm{pf}} P / N_{\mathrm{t}}}{N_{0}+N_{\mathrm{pf}} P / N_{\mathrm{t}}}\right)^{-1}}\left[\hat{\mathbf{H}}_{k, 1}^{\mathrm{f}}, \ldots, \hat{\mathbf{H}}_{k, K}^{\mathrm{f}}\right] \boldsymbol{\Theta}_{k}
$$

such that $\mathbb{E}\left[\operatorname{tr}\left(\mathbf{X}_{k} \mathbf{X}_{k}^{H}\right)\right]=N_{\mathrm{fb}} P_{\mathrm{r}}$. The approach in [18] can be followed to derive the error due to forward channel estimation, reverse channel estimation, and analog CSI feedback. The concatenated matrix of received feedback symbols by all the transmitters is

$$
\begin{aligned}
\mathbf{Y}= & \sqrt{\frac{N_{\mathrm{fb}} P_{\mathrm{r}}}{K N_{\mathrm{t}} N_{\mathrm{r}}}\left(\frac{N_{\mathrm{pf}} P / N_{\mathrm{t}}}{N_{0}+N_{\mathrm{pf}} P / N_{\mathrm{t}}}\right)^{-1}} \\
& \times \sum_{k=1}^{K}\left[\begin{array}{c}
\mathbf{H}_{k, 1}^{\mathrm{r}} \\
\vdots \\
\mathbf{H}_{k, K}^{\mathrm{r}}
\end{array}\right]\left[\begin{array}{lll}
\hat{\mathbf{H}}_{k, 1}^{\mathrm{f}} & \cdots & \hat{\mathbf{H}}_{k, K}^{\mathrm{f}}
\end{array}\right] \boldsymbol{\Theta}_{k}+\mathbf{Z}
\end{aligned}
$$

where $\mathbf{Z} \in \mathbb{C}^{K N_{\mathrm{t}} \times N_{\mathrm{fb}}}$ is the noise matrix. The channels $\mathbf{H}_{k, j}$, estimated and fed back by receiver $k \forall j$, are re-estimated at transmitter $k$ by post-multiplying $\mathbf{Y}$ by $\boldsymbol{\Theta}_{k}^{H}$. Borrowing the derivations in [18, Sec. IV], which are summarized for the sake of completeness in Appendix A, the final expression for the variance of the channel estimation error is

$$
\sigma_{\tilde{\mathbf{H}}}^{2}=\frac{N_{\mathrm{t}}}{\rho d N_{\mathrm{pf}}}+\frac{1}{\rho d\left(K N_{\mathrm{t}}-N_{\mathrm{r}}\right)}\left(\frac{N_{\mathrm{r}}^{2}}{\beta N_{\mathrm{pr}}}+\frac{K N_{\mathrm{t}} N_{\mathrm{r}}}{\beta N_{\mathrm{fb}}}\right) .
$$

We note that the channel estimates $\hat{\mathbf{H}}_{k, j}$ available at the transmitters are no longer complex Gaussian due to the fading introduced by the feedback channel. However, $\hat{\mathbf{H}}_{k, j}$ can be approximated as complex Gaussian with variance $1-\sigma_{\tilde{\mathbf{H}}}^{2}$. This was verified in [18], where it was shown that the effect of this approximation is rather negligible.

It is important to realize that the concatenated $K N_{\mathrm{t}} \times N_{\mathrm{fb}}$ matrix $\mathbf{Y}$ in (29) can be constructed only if the transmitters share their locally observed rows; then, the computation of the IA precoders can be centralized as described in Section I. This is in principle feasible in the downlink, where the transmitters are embodied by base stations connected through the backhaul, and such is the focus of our analysis. In the uplink, or in downlink settings where CSI sharing could not be implemented, one would have to resort to less desirable alternatives. The 
first such alternative would be distributed IA with over-the-air iterations, which would incur additional signaling overhead. The second alternative would be the closed-form computation of the precoders based on estimates of all the forward channels obtained from observing the $N_{\mathrm{t}} \times N_{\mathrm{fb}}$ feedback matrix locally. However, this second alternative would only be feasible for those configurations for which precoder closed forms are indeed available. Moreover, since in this approach every transmitter would observe the feedback matrix through a different reverse fading channel, each transmitter would have a differently perturbed estimate of the forward channels; this would cause an additional (small) performance loss [13].

4) Forward Dedicated Pilots: The precoder matrix $\hat{\mathbf{V}}_{k}$ at transmitter $k$, computed using the estimates $\hat{\mathbf{H}}_{k, j} \forall j \neq k$, is unknown at receiver $k$. An additional round of dedicated pilots is required once the transmitters have computed their precoders in order to enable each receiver $k$ to learn the precoded channels. Precisely, each transmitter $k$ transmits orthogonal pilots along its precoder $\hat{\mathbf{V}}_{k} \in \mathbb{C}^{N_{\mathrm{t}} \times d}$ over $N_{\mathrm{pd}} \geq K d$ symbols. The observed signal at receiver $k$ is then

$$
\mathbf{Y}_{k}=\sqrt{\frac{N_{\mathrm{pd}} P}{d}} \sum_{j=1}^{K} \mathbf{H}_{k, j} \hat{\mathbf{V}}_{j} \boldsymbol{\Omega}_{j}+\mathbf{Z}_{k}
$$

where $\boldsymbol{\Omega}_{j}$ is the dedicated pilot sequence matrix at transmitter $j$.

Each receiver $k$ estimates its desired precoded channel $\mathbf{A}_{k, k}=\mathbf{H}_{k, k} \hat{\mathbf{V}}_{k}$. Since $\hat{\mathbf{V}}_{k}$ is unitary and independent of $\mathbf{H}_{k, k}$, it follows that $\mathbf{A}_{k, k}$ is complex Gaussian. The MMSE estimate of $\mathbf{A}_{k, k}$ is then

$$
\hat{\mathbf{A}}_{k, k}=\frac{\sqrt{\frac{N_{\mathrm{pd}} P}{d}}}{N_{0}+\frac{N_{\mathrm{pd}} P}{d}} \mathbf{Y}_{k} \boldsymbol{\Omega}_{k}^{H}
$$

and the corresponding error $\tilde{\mathbf{A}}_{k, k}=\mathbf{A}_{k, k}-\hat{\mathbf{A}}_{k, k}$ is complex Gaussian with variance

$$
\begin{aligned}
\sigma_{\tilde{\mathbf{A}}}^{2} & =\frac{N_{0}}{N_{0}+N_{\mathrm{pd}} P / d} \\
& =\frac{1}{1+N_{\mathrm{pd}} \rho}
\end{aligned}
$$

wheras $\hat{\mathbf{A}}_{k, k}$ is complex Gaussian with variance $1-\sigma_{\tilde{\mathbf{A}}}^{2}$.

Plugging (30) and (34) into (20), the effective average perstream SINR in (20) specializes into (35) (given at the top of the next page), which is a function of: the forward link common pilot overhead $N_{\mathrm{pf}}$, the reverse link common pilot overhead $N_{\mathrm{pr}}$, the feedback overhead $N_{\mathrm{fb}}$, the dedicated pilot overhead $N_{\mathrm{pd}}$, the average SNR per stream $\rho$, the ratio of reverse and forward link powers $\beta$, the DoF per user $d$, the time-domain fading correlation $\epsilon[\cdot]$, the number of users $K$, and the number of transmit antennas $N_{\mathrm{t}}$.

\section{B. $T D D$}

With TDD, the same frequency band is shared by the forward and reverse links and thus the corresponding overthe-air channels are transposed versions of each other. Then, with careful calibration, the analog CSI feedback phase is not required. Recall that the reverse channel from receiver $k$ to transmitter $j$ is $\mathbf{H}_{j, k}^{\mathrm{r}}=\mathbf{H}_{k, j}^{T}$ where $\mathbf{H}_{k, j}$ is the forward channel from transmitter $j$ to receiver $k$. Further recalling the reverse channel estimate and the corresponding error matrix as $\hat{\mathbf{H}}_{k, j}^{\mathrm{r}}$ and $\tilde{\mathbf{H}}_{k, j}^{\mathrm{r}}$, with entries distributed according to (26) and (27), respectively, transmitter $k$ computes its forward channel estimate $\hat{\mathbf{H}}_{k, j}$ as simply the transpose of $\hat{\mathbf{H}}_{j, k}^{r}$ and the variance of the channel estimation error is

$$
\begin{aligned}
\sigma_{\tilde{\mathbf{H}}}^{2} & =\frac{N_{0}}{N_{0}+\frac{N_{\mathrm{pr}} P_{\mathrm{r}}}{N_{\mathrm{r}}}} \\
& =\frac{1}{1+\frac{N_{\mathrm{pr}}}{N_{\mathrm{r}}} \beta \rho d} .
\end{aligned}
$$

Utilizing (34) and (37) in (20), the effective average per-stream SINR can be derived for TDD as

$$
\rho_{\text {eff }}[n]=\frac{\epsilon[n]^{2} \rho\left(1-\frac{1}{1+\rho N_{\mathrm{pd}}}\right)}{1+\rho K d+\epsilon[n]^{2} \rho d\left(-K+\frac{1}{1+N_{\mathrm{pd}} \rho}+\frac{(K-1) N_{\mathrm{r}}}{N_{\mathrm{r}}+\beta \rho d N_{\mathrm{pr}}}\right)}
$$

\section{Optimum Pilot Overhead And IA Update INTERVAL}

\section{A. FDD}

The effective average sum mutual information is the solution to

$$
\max _{N_{\mathrm{pf}}, N_{\mathrm{pr}}, N_{\mathrm{fb}}, N_{\mathrm{pd}}, N_{\mathrm{b}}} \frac{1}{N_{\mathrm{b}}} \sum_{n=1}^{N_{\mathrm{b}}-N_{\mathrm{p}}} \bar{I}_{\text {sum }}[n]
$$

subject to

$$
\begin{aligned}
& N_{\mathrm{pf}} \geq K N_{\mathrm{t}} \\
& N_{\mathrm{pr}} \geq K N_{\mathrm{r}} \\
& N_{\mathrm{fb}} \geq K^{2} N_{\mathrm{t}} \\
& N_{\mathrm{pd}} \geq K d
\end{aligned}
$$

with $\bar{I}_{\text {sum }}[\cdot]$ given in (22) and $\rho_{\text {eff }}[\cdot]$ given in (35). The optimum value of a given variable is henceforth indicated with a superscript $(\cdot)^{\star}$, e.g., $N_{\mathrm{pf}}^{\star}$. With coding spanning enough update intervals, (39) signifies the achievable average sum spectral efficiency.

1) Optimization of $N_{\mathrm{b}}$ with Fixed Overhead: Increasing $N_{\mathrm{pf}}, N_{\mathrm{pr}}, N_{\mathrm{fb}}$ and $N_{\mathrm{pd}}$ improves the CSI accuracy yielding a higher effective SINR at the expense of a higher overhead. While the improvement in spectral efficiency associated with the SINR is ultimately logarithmic, the decrease associated with the overhead is always linear. Thus, at sufficiently high SNR the optimum overhead is bound to take the minimum value corresponding to $N_{\mathrm{pf}}^{\star}=K N_{\mathrm{t}}, N_{\mathrm{pr}}^{\star}=K N_{\mathrm{r}}, N_{\mathrm{fb}}^{\star}=$ $K^{2} N_{\mathrm{t}}, N_{\mathrm{pd}}^{\star}=K d$ and $N_{\mathrm{p}}^{\star}=K\left((K+1) N_{\mathrm{t}}+N_{\mathrm{r}}+d\right)$. Utilizing these values, which for the reader's convenience are summarized in Table I, the corresponding effective average per-stream SINR becomes (44), and the optimization in (39) simplifies to $\max _{N_{\mathrm{b}}} f\left(N_{\mathrm{b}}\right)$ with

$$
f\left(N_{\mathrm{b}}\right)=\frac{1}{N_{\mathrm{b}}} \sum_{n=1}^{N_{\mathrm{b}}-N_{\mathrm{p}}^{\star}} \bar{I}_{\mathrm{sum}}[n] .
$$




$$
\rho_{\mathrm{eff}}[n]=\frac{\epsilon[n]^{2} \rho\left(1-\frac{1}{1+\rho N_{\mathrm{pd}}}\right)}{1+\rho K d+\epsilon[n]^{2}\left(\frac{(K-1) N_{\mathrm{t}}}{N_{\mathrm{pf}}}+(K-1)\left(\frac{N_{\mathrm{rr}}^{2}+\frac{K N_{\mathrm{t}} N_{\mathrm{r}}}{\beta N_{\mathrm{fb}}}}{\left(K N_{\mathrm{t}}-N_{\mathrm{r}}\right)}\right)+\rho d\left(-K+\frac{1}{1+\rho N_{\mathrm{pd}}}\right)\right)}
$$

$$
\rho_{\text {eff }}[n]=\frac{\epsilon[n]^{2} \rho^{2} K d}{(1+\rho K d)\left(1+\rho K d+\epsilon[n]^{2}\left(1+\frac{2(K-1) N_{\mathrm{r}}}{\beta K^{2} N_{\mathrm{t}}-\beta K N_{\mathrm{r}}}-\rho K d-\frac{1}{K+\rho K^{2} d}\right)\right)}
$$

We are thus faced with an integer problem. With a continuous relaxation thereof, the argument of the optimization becomes

$$
f\left(N_{\mathrm{b}}\right)=\frac{1}{N_{\mathrm{b}}} \int_{1}^{N_{\mathrm{b}}-N_{\mathrm{p}}^{\star}} \bar{I}_{\text {sum }}[n] \mathrm{d} n
$$

with derivative

$$
\dot{f}\left(N_{\mathrm{b}}\right)=-\frac{1}{N_{\mathrm{b}}} f\left(N_{\mathrm{b}}\right)+\frac{1}{N_{\mathrm{b}}} \bar{I}_{\text {sum }}\left[N_{\mathrm{b}}-N_{\mathrm{p}}^{\star}\right] .
$$

It can be easily verified that $f($.$) has a unique stationary$ point and that, since $\bar{I}_{\text {sum }}[n]$ is a decreasing function of $n$, the second derivative at this point is negative [20, App. II]. Thus, its global maximum can be found by solving

$$
-\frac{1}{N_{\mathrm{b}}} f\left(N_{\mathrm{b}}\right)+\frac{1}{N_{\mathrm{b}}} \bar{I}_{\text {sum }}\left[N_{\mathrm{b}}-N_{\mathrm{p}}^{\star}\right]=0 .
$$

This leads to the condition $f\left(N_{\mathrm{b}}\right)=\bar{I}_{\mathrm{sum}}\left[N_{\mathrm{b}}-N_{\mathrm{p}}^{\star}\right]$ or, more explicitly, to the fixed-point equation

$$
\frac{1}{N_{\mathrm{b}}} \sum_{n=1}^{N_{\mathrm{b}}-N_{\mathrm{p}}^{\star}} \bar{I}_{\text {sum }}[n]=\bar{I}_{\text {sum }}\left[N_{\mathrm{b}}-N_{\mathrm{p}}^{\star}\right]
$$

where, with all the overheads at their respective minima, $N_{\mathrm{p}}^{\star}=$ $K\left((K+1) N_{\mathrm{t}}+N_{\mathrm{r}}+d\right)$. Given the continuous relaxation used to reach (49), it is necessary to test the two nearest integer neighbors to its real-valued solution for $N_{\mathrm{b}}$ in order to identify $N_{\mathrm{b}}^{\star}$.

TABLE I

MINIMUM PILOT OVERHEAD EXPRESSIONS FOR IA-FDD AND IA-TDD.

\begin{tabular}{|l||l|l|}
\hline & IA-FDD & IA-TDD \\
\hline \hline$N_{\mathrm{pf}}$ & $K N_{\mathrm{t}}$ & $K N_{\mathrm{t}}$ \\
\hline$N_{\mathrm{pr}}$ & $K N_{\mathrm{r}}$ & $K N_{\mathrm{r}}$ \\
\hline$N_{\mathrm{fb}}$ & $K^{2} N_{\mathrm{t}}$ & - \\
\hline$N_{\mathrm{pd}}$ & $K d$ & $K d$ \\
\hline$N_{\mathrm{p}}$ & $K\left((K+1) N_{\mathrm{t}}+N_{\mathrm{r}}+d\right)$ & $K\left(N_{\mathrm{t}}+N_{\mathrm{r}}+d\right)$ \\
\hline
\end{tabular}

2) High-SNR Optimization: For certain time-domain correlation functions, solving (49) is numerically challenging because $\bar{I}_{\text {sum }}[\cdot]$ in (22) contains an exponential integral. At high SNR, however, an alternative to (22) can be obtained by expanding (21) into [31]

$$
\bar{I}_{\text {sum }}[n]=K d\left(\log _{2} \rho_{\text {eff }}[n]-\mathcal{L}_{\infty}[n]\right)+o(1)
$$

where $\mathcal{L}_{\infty}=\mathbb{E}\left[\log _{2}\left(\left|\mathbf{h}_{\text {eff }, \ell}\right|^{2}\right)\right]$. Since $\mathbf{h}_{\text {eff }, \ell}$ is complex Gaussian with unit variance, $\left|\mathbf{h}_{\text {eff }, \ell}\right|^{2}$ is exponentially distributed with mean one and with $\mathcal{L}_{\infty}=\mathcal{C} \log _{2} e$ where
$\mathcal{C} \approx 0.5772$ is the Euler-Mascheroni constant. Plugging the above expansion into (49), we obtain the far simpler fixedpoint equation

$$
N_{\mathrm{b}}^{\star} \log _{2} \rho_{\text {eff }}\left[N_{\mathrm{b}}^{\star}-N_{\mathrm{p}}^{\star}\right]-\sum_{n=1}^{N_{\mathrm{b}}^{\star}-N_{\mathrm{p}}^{\star}} \log _{2} \rho_{\text {eff }}[n]=N_{\mathrm{p}}^{\star} \mathcal{L}_{\infty}+o(1)
$$

where, recall, $N_{\mathrm{p}}^{\star}=K\left((K+1) N_{\mathrm{t}}+N_{\mathrm{r}}+d\right)$. From (51), $N_{\mathrm{b}}^{\star}$ can be found conveniently down to the $o(1)$ term-which vanishes with growing SNR. Again, the two closest integer neighbors to the solution should be tested to overcome the effects of the continuous relaxation.

\section{B. $T D D$}

The problem formulation for TDD is similar to the one for FDD except for the absence of the analog CSI feedback stage. Then, the effective average sum spectral efficiency is optimized over $N_{\mathrm{pf}}, N_{\mathrm{pr}}, N_{\mathrm{pd}}$ and $N_{\mathrm{b}}$ with $N_{\mathrm{pf}} \geq K N_{\mathrm{t}}, N_{\mathrm{pr}} \geq$ $K N_{\mathrm{r}}$ and $N_{\mathrm{pd}} \geq K d$.

1) Optimization of $N_{\mathrm{b}}$ with Fixed Overheads: Leveraging the approach in Section V-A1, the minimum overhead in TDD is $N_{\mathrm{p}}^{\star}=K\left(N_{\mathrm{t}}+N_{\mathrm{r}}+d\right)$ while the corresponding effective average per-stream SINR in (38) reduces to (52) (given at the top of the next page). With the overhead at its minimum value, the optimum update interval $N_{\mathrm{b}}^{\star}$ at an arbitrary SNR can be computed as the solution of (49) while the corresponding highSNR optimization solution can be found by solving (51). Once more, in both cases, the two closest integer neighbors to the real-valued solution should be tested.

\section{Numerical Results}

In order to generate some numerical results, we invoke the standard Clarke-Jakes correlation function $\epsilon[n]=$ $J_{0}\left(2 \pi f_{\mathrm{m}} n T_{\mathrm{s}}\right)$ where $T_{\mathrm{s}}$ is the symbol period and $f_{\mathrm{m}}$ is the maximum Doppler shift (in $\mathrm{Hz}$ ). However, because of the oscillatory nature of $J_{0}(\cdot)$, such $\epsilon[n]$ is not a monotonically decreasing function of $n$ and thus it does not strictly satisfy the conditions of our analysis. By replacing $J_{0}(\cdot)$ by its expansion

$$
J_{0}(z)=1-\frac{z^{2}}{4}+\frac{z^{4}}{64}+o\left(z^{4}\right)
$$

we obtain a monotonically decreasing correlation function that mimics very precisely the Clarke-Jakes behavior in the range of interest, for all reasonable values of the parameters' space. 


$$
\rho_{\text {eff }}[n]=\frac{\epsilon[n]^{2} \rho^{2} K d}{(1+\rho K d)\left(1+\rho d\left(K+\epsilon[n]^{2}\left(-K+\frac{1}{1+\rho K d}+\frac{K-1}{1+\beta \rho K d}\right)\right)\right)}
$$

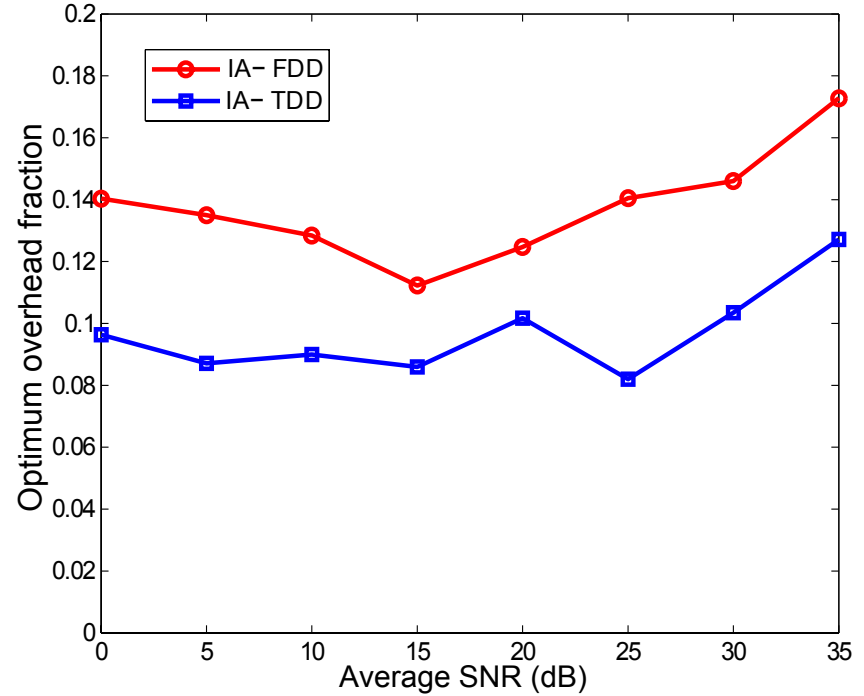

Fig. 3. Optimum overhead fraction v. SNR for IA with $K=3, d=1$, $N_{\mathrm{t}}=N_{\mathrm{r}}=2, f_{\mathrm{m}}=10 \mathrm{~Hz}$ and $T_{\mathrm{s}}=5.5 \mu \mathrm{s}$.

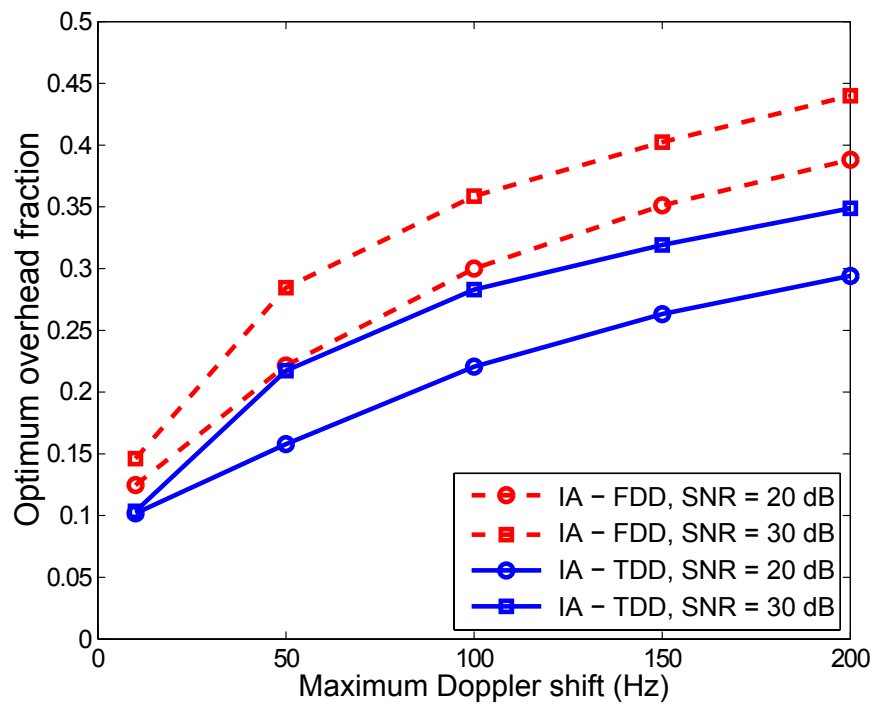

Fig. 4. Optimum overhead fraction v. maximum Doppler shift at $\mathrm{SNR}=20$ $\mathrm{dB}$ and $\mathrm{SNR}=30 \mathrm{~dB}$ with $K=3, d=1, N_{\mathrm{t}}=N_{\mathrm{r}}=2$ and $T_{\mathrm{s}}=5.5 \mu \mathrm{s}$.

The degradation associated with time selectivity is bound to subside as $T_{\mathrm{s}}$ shrinks, but, if $B_{\mathrm{c}}$ denotes the coherence bandwidth, $T_{\mathrm{s}}$ cannot fall below (roughly) $1 / B_{\mathrm{c}}$ since otherwise the flat-fading condition in the formulation would be violated. For our results, we choose a very favorable value for IA and set $T_{\mathrm{s}}=1 / B_{\mathrm{c}}$ with $B_{\mathrm{c}}=180 \mathrm{kHz}$; this corresponds to one LTE signaling resource block [32]. Then, $T_{\mathrm{s}}=5.5 \mu \mathrm{s}$. Furthermore, for the examples that follow, unless otherwise stated, $K=3$, $N_{\mathrm{t}}=N_{\mathrm{r}}=2, d=1$ and $P_{\mathrm{r}}=P$.

Figure 3 shows, as function of the SNR, the optimal pilot overhead fraction, $N_{\mathrm{p}}^{\star} / N_{\mathrm{b}}^{\star}$, obtained by solving (39) numeri-

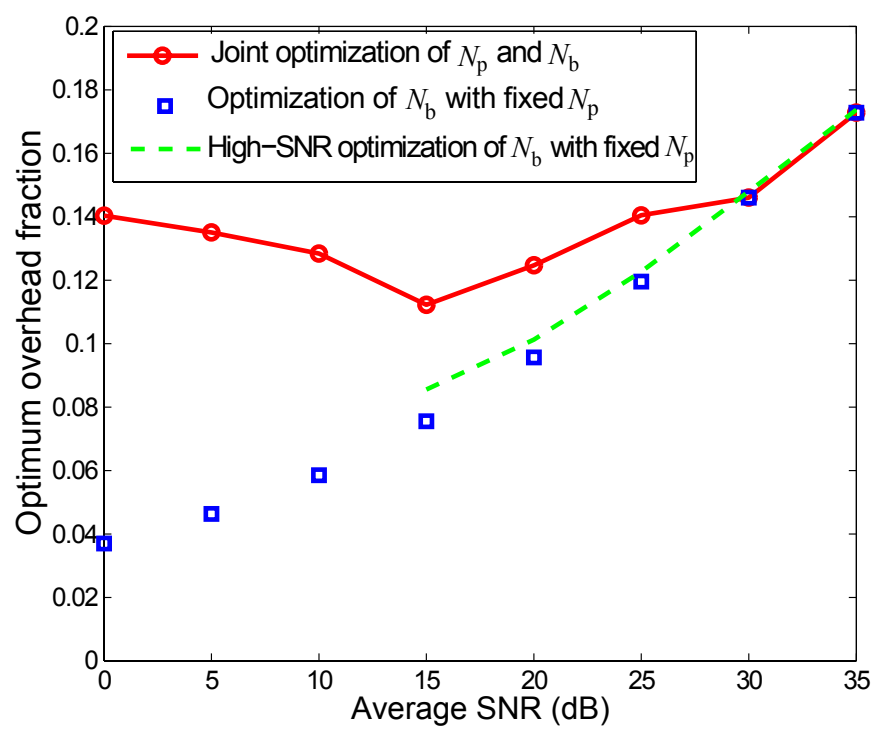

Fig. 5. Optimum overhead fraction v. SNR for IA-FDD with $K=3, d=1$, $N_{\mathrm{t}}=N_{\mathrm{r}}=2, f_{\mathrm{m}}=10 \mathrm{~Hz}$ and $T_{\mathrm{s}}=5.5 \mu \mathrm{s}$.

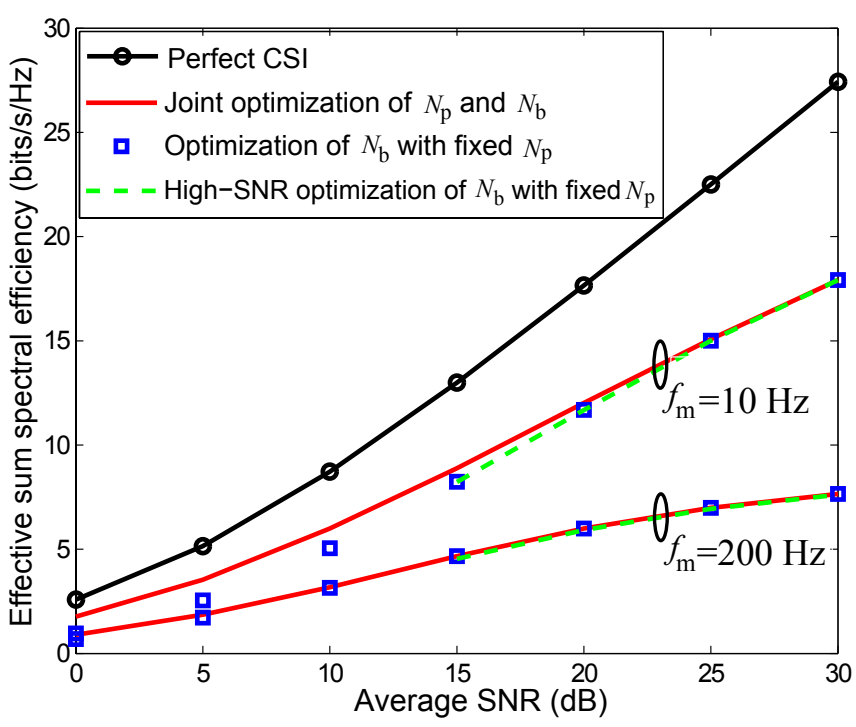

Fig. 6. Effective sum spectral efficiency v. SNR for IA-FDD at $f_{\mathrm{m}}=10$ $\mathrm{Hz}$ and $f_{\mathrm{m}}=200 \mathrm{~Hz}$ with $K=3, d=1, N_{\mathrm{t}}=N_{\mathrm{r}}=2$, and $T_{\mathrm{s}}=5.5 \mu \mathrm{s}$.

cally for both FDD and TDD. The maximum Doppler shift is $f_{\mathrm{m}}=10 \mathrm{~Hz}$, which corresponds to a pedestrian velocity of 5 $\mathrm{Km} / \mathrm{h}$ at a carrier frequency of $2 \mathrm{GHz}$. The lack of smoothness in the curves is a direct consequence of the integer nature of the problem. The optimum overhead is higher with FDD due to the need for explicit CSI feedback, as one would expect.

For a different perspective, Fig. 4 presents the optimal pilot overhead fraction, $N_{\mathrm{p}}^{\star} / N_{\mathrm{b}}^{\star}$, as function of $f_{\mathrm{m}}$. As one would again expect, $N_{\mathrm{b}}^{\star}$ declines as $f_{\mathrm{m}}$ increases, and the overhead fraction worsens. Less foreseeable, and thus a much 


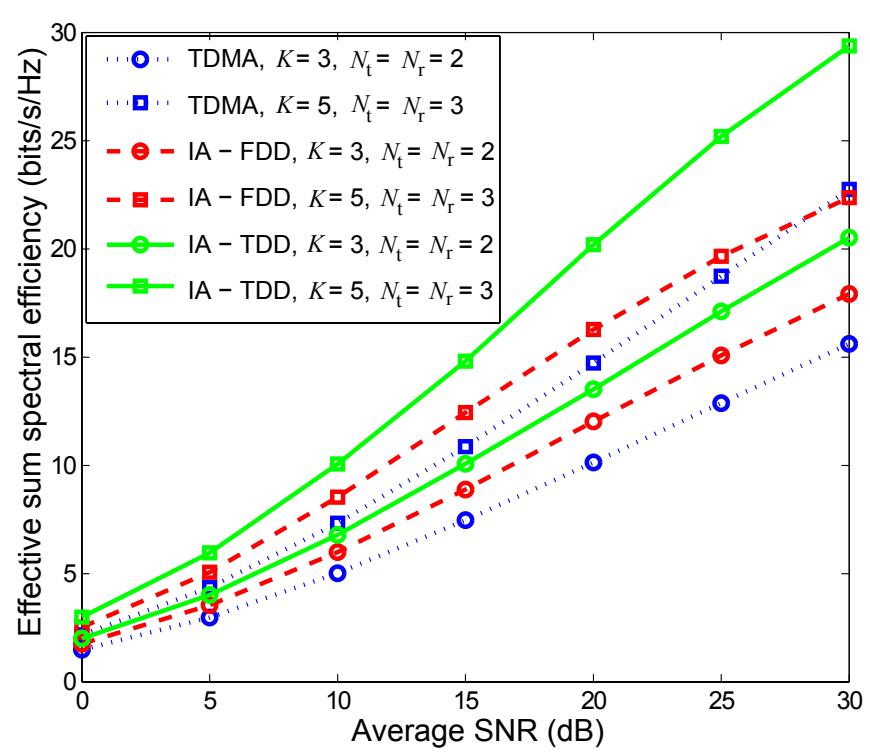

Fig. 7. Effective sum spectral efficiency v. SNR for IA and TDMA with $K=3$ and $K=5, f_{\mathrm{m}}=10 \mathrm{~Hz}$ and $T_{\mathrm{s}}=5.5 \mu \mathrm{s}$.

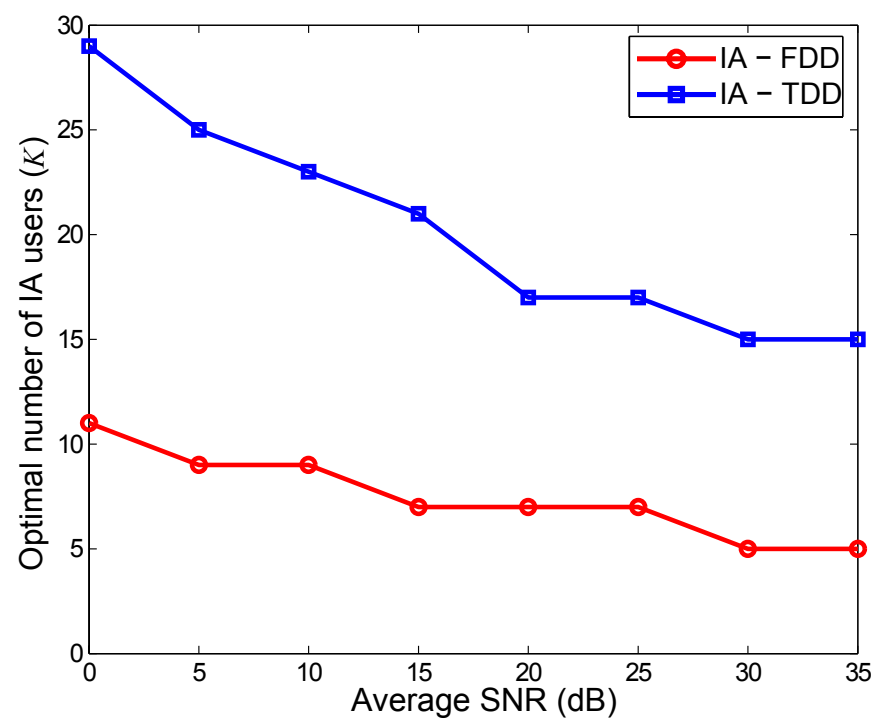

Fig. 8. Optimal number of IA users (corresponding to maximum effective sum spectral efficiency) v. SNR for IA-FDD and IA-TDD with $d=1, f_{\mathrm{m}}=$ $10 \mathrm{~Hz}$ and $T_{\mathrm{s}}=5.5 \mu \mathrm{s}$.

more interesting insight, is the dependence of the overhead fraction on the SNR: in contrast with single-user MIMO, where the overhead fraction shrinks with a growing SNR [21], in IA it increases, the reason being that with less noise the performance becomes more sensitive to Doppler-induced precoder deviations and this sensitivity triggers more frequent precoder updates, i.e., it also reduces $N_{\mathrm{b}}^{\star}$.

Figure 5 compares the optimal pilot overhead fraction obtained by solving (39) numerically against the solution $K\left((K+2) N_{\mathrm{t}}+d\right) / N_{\mathrm{b}}^{\star}$ where $N_{\mathrm{b}}^{\star}$ is derived from (49), and further against the solution provided by (51). The maximum Doppler shift is $f_{\mathrm{m}}=10 \mathrm{~Hz}$. As anticipated, all the results agree at high SNR, once $N_{\mathrm{p}}^{\star}$ has reached its minimum value. The sustained increase of the overhead with the SNR is ex-

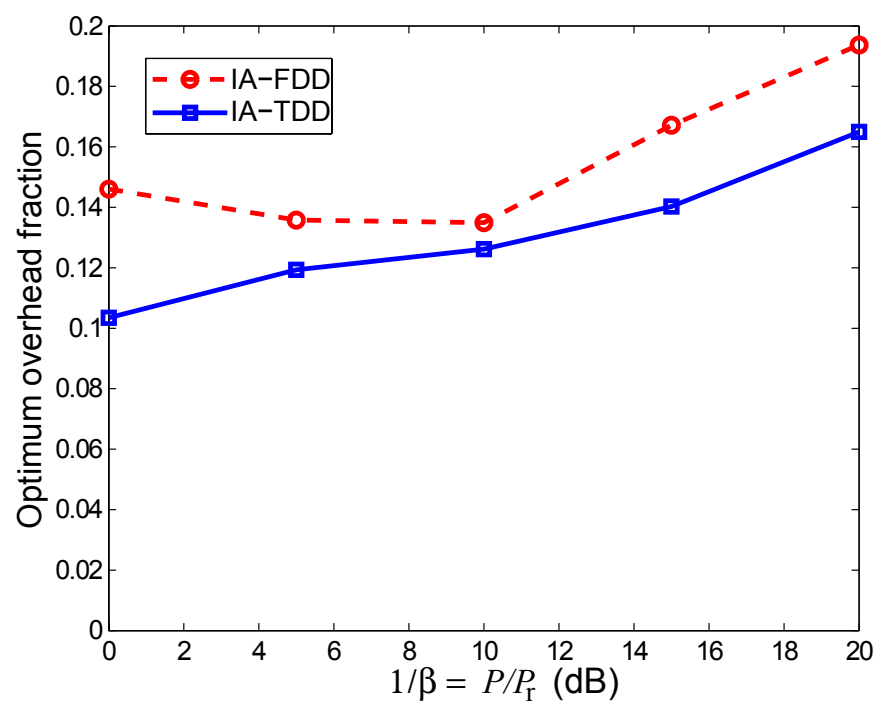

Fig. 9. Optimum overhead fraction v. $1 / \beta$ for IA-FDD and IA-TDD with $K=3, d=1, N_{\mathrm{t}}=N_{\mathrm{r}}=2$, forward link average SNR $=30 \mathrm{~dB}, f_{\mathrm{m}}=10$ $\mathrm{Hz}$ and $T_{\mathrm{s}}=5.5 \mu \mathrm{s}$.

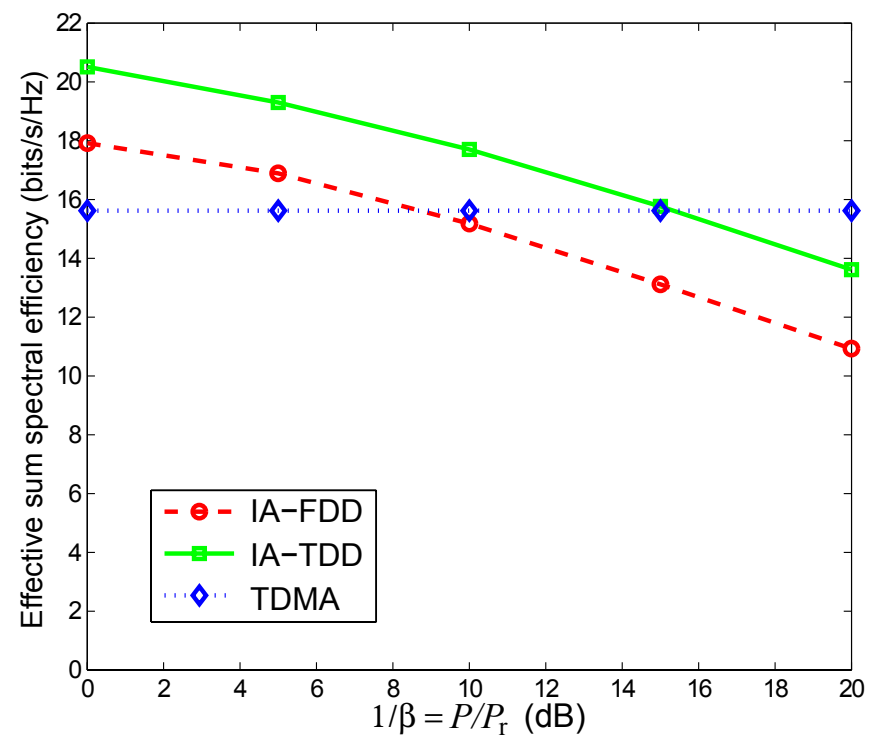

Fig. 10. Effective sum spectral efficiency v. $1 / \beta$ for IA-FDD, IA-TDD and TDMA with $K=3, d=1, N_{\mathrm{t}}=N_{\mathrm{r}}=2$, forward link average SNR $=30$ $\mathrm{dB}, f_{\mathrm{m}}=10 \mathrm{~Hz}$ and $T_{\mathrm{s}}=5.5 \mu \mathrm{s}$.

plained by the rising sensitivity to time selectivity: the channel estimation becomes more precise with the SNR, rendering the misalignment caused by selectivity proportionally more acute; this induces more frequent IA updates, i.e., a smaller $N_{\mathrm{b}}^{\star}$.

Turning now our attention to the effective sum spectral efficiency, it is shown in Fig. 6 as function of the SNR for $f_{\mathrm{m}}=10 \mathrm{~Hz}$ and $f_{\mathrm{m}}=200 \mathrm{~Hz}$, respectively corresponding to pedestrian and vehicular velocities at $2 \mathrm{GHz}$. Specifically, FDD is considered in these results.

Figure 7 compares the effective sum spectral efficiencies of IA-FDD, IA-TDD and TDMA as function of SNR in a pedestrian scenario. Here, settings with $K=3$ and $K=5$ users are contrasted. In both cases, IA-FDD and IA-TDD are seen to outperform TDMA, although with FDD the improvement 
is minor. In vehicular scenarios, the IA advantage vanishes entirely regardless of whether TDD or FDD is employed, confirming the widespread conjecture that IA is not applicable to these scenarios. For example, with $K=3$ and $\mathrm{SNR}=20$ $\mathrm{dB}$, we have observed that the IA-FDD advantage vanishes beyond $f_{\mathrm{m}}=100 \mathrm{~Hz}$ while the IA-TDD advantage vanishes beyond $f_{\mathrm{m}}=200 \mathrm{~Hz}$. In the remainder we therefore focus exclusively on pedestrian settings.

After having exemplified the IA performance with typical cooperation sizes of $K=3$ and $K=5$, it is reasonable to wonder the extent to which the observations made for these values are general. Short of repeating the examples for all possible values of $K$, the best way to address this question is by assessing the optimum value of $K$ as function of the parameters of interest, chiefly the SNR. The result of this assessment is precisely what Fig. 8 presents: the best possible value of $K$ as function of SNR in a pedestrian scenario $\left(f_{\mathrm{m}}=10 \mathrm{~Hz}\right)$. The numbers of antennas are chosen to satisfy the IA feasibility condition in (6) for $d=1$. As can be seen, $K$ declines progressively and, at $30 \mathrm{~dB}$, it equals the value of $K=5$ utilized in some of the earlier examples.

Finally, we turn our attention to the impact of asymmetric forward and reverse channel strengths. Both IA-FDD and IATDD are considered, with a forward link average SNR of 30 dB. Figure 9 shows the variation of the optimum overhead fraction with respect to $1 / \beta=P / P_{\mathrm{r}}$. Figure 10 quantifies the effective sum spectral efficiency as a function of $1 / \beta$ and compares it against the TDMA spectral efficiency at the same SNR. As the reverse link weakens with respect to the forward link, the performance of IA progressively degrades while that of TDMA remains unaffected. Hence, IA becomes unappealing in the presence of a sufficiently weak reverse link.

\section{CONCLUSION}

In this paper, the optimum pilot overhead and IA update interval in a $K$-user MIMO interference channel have been obtained as solutions to an optimization, which at high SNR adopts a particularly tractable form. This solution, in turn, allows for a straight computation of the spectral efficiency achievable by IA in time-selective fading channels. Variations of the formulation for both FDD and TDD have been given.

A number of numerical examples have been presented, corresponding to typical operating conditions in terms of SNR, fading coherence, and numbers of antennas. Through these examples, it has been observed that, with fading selectivity and pilot-assisted channel estimation explicitly incorporated, IA loses all its advantage over TDMA at vehicular speeds, but remains somewhat superior at pedestrian speeds. The improvement is minor in the case of FDD, and more significant in the case of TDD.

Tempered by the considerations made in [33]-[35], chiefly that the insights obtained from the $K$-user interference channel apply to large wireless networks only within a certain SNR range, IA is seen to retain the potential to play some role in the management of interference in pedestrian-oriented TDD wireless systems; that role, however, might be largely circumscribed to cell-edge users for which the interference channel is a reasonable model. In FDD and/or vehicular-oriented systems, conversely, the various overheads nullify the gains that IA would provide over TDMA if perfect CSI were available at no cost.

To be sure, maximum-SINR solutions would perform somewhat better than IA at intermediate SNR levels, but hardly in the high-SNR regime where intercell interference becomes the dominating impairment.

We also note that techniques that can be leveraged to improve pilot-assisted communication in single-user communication, e.g., channel interpolation or data-aided channel tracking [28], cannot be directly applied in IA because the precoders and receive filters must be jointly computed. If, for instance, the precoders were to be modified at any intermediate point within the update intervals, new dedicated pilots would have to be transmitted to enable proper retuning of the receivers; this would increase the overheads even further. What could be done, respecting the regular IA update approach followed in this paper and avoiding a further increase in the overheads, is to compute the precoders and receivers by considering not only the set of pilot observations preceding the current update interval, but also older ones. We have explored this possibility and observed that, because of the fading decorrelation across multiple update intervals, the corresponding improvements in spectral efficiency are minute.

Finally, we hasten to emphasize that the performance of IA would further degrade should a distributed implementation be necessary, as this would entail a substantial number of over-the-air iterations (each involving forward and reverse pilot transmissions) within every update interval. The range of operational conditions where IA may be enticing would then be squeezed down very substantially and it might be the case that IA no longer retains any potential to play a role in wireless interference management.

\section{APPENDiX A}

PROOF OF (30)

Post-multiplying $\mathbf{Y}$ by $\boldsymbol{\Theta}_{k}^{H}$, we obtain

$$
\begin{aligned}
\mathbf{Y} \Theta_{k}^{H}= & \sqrt{\frac{N_{\mathrm{fb}} P_{\mathrm{r}}}{K N_{\mathrm{t}} N_{\mathrm{r}}}\left(\frac{N_{\mathrm{pf}} P / N_{\mathrm{t}}}{N_{0}+N_{\mathrm{pf}} P / N_{\mathrm{t}}}\right)^{-1}} \\
& \times \underbrace{\left[\begin{array}{c}
\mathbf{H}_{k, 1}^{\mathrm{r}} \\
\vdots \\
\mathbf{H}_{k, K}^{\mathrm{r}}
\end{array}\right]}_{\mathbf{H}_{k}^{\mathrm{r}}}\left[\begin{array}{lll}
\hat{\mathbf{H}}_{k, 1}^{\mathrm{f}} & \cdots & \hat{\mathbf{H}}_{k, K}^{\mathrm{f}}
\end{array}\right]+\mathbf{Z} \Theta_{k}^{H} .
\end{aligned}
$$

Given the condition $K N_{\mathrm{t}} \geq N_{\mathrm{r}}$, the application of the orthogonality principle yields the MMSE estimate of the forward channels corresponding to receiver $k$ as

$$
\begin{aligned}
\hat{\mathbf{H}}_{k}= & \sqrt{\frac{K N_{\mathrm{t}} N_{\mathrm{r}}}{N_{\mathrm{fb}} P_{\mathrm{r}}}\left(\frac{N_{\mathrm{pf}} P / N_{\mathrm{t}}}{N_{0}+N_{\mathrm{pf}} P / N_{\mathrm{t}}}\right)^{-1}} \\
& \cdot\left(\hat{\mathbf{H}}_{k}^{\mathrm{r} H} \hat{\mathbf{H}}_{k}^{\mathrm{r}}+\zeta_{1} \hat{\mathbf{H}}_{k}^{\mathrm{r} H} \hat{\mathbf{H}}_{k}^{\mathrm{r}}+\zeta_{2} I_{N_{\mathrm{r}}}\right)^{-1} \hat{\mathbf{H}}_{k}^{\mathrm{r}} \mathbf{Y} \Theta_{k}^{H}
\end{aligned}
$$


where $\hat{\mathbf{H}}_{k}=\left[\hat{\mathbf{H}}_{k, 1}, \cdots, \hat{\mathbf{H}}_{k, K}\right]$ represents the concatenated estimate of $\mathbf{H}_{k}=\left[\mathbf{H}_{k, 1}, \cdots, \mathbf{H}_{k, K}\right]$ while

$$
\zeta_{1}=\frac{N_{\mathrm{t}} N_{0}}{P N_{\mathrm{pf}}}
$$

and

$$
\zeta_{2}=\left(1+\frac{N_{\mathrm{t}} N_{0}}{P N_{\mathrm{pf}}}\right)\left(\frac{N_{0} K N_{\mathrm{t}} N_{\mathrm{r}}}{N_{\mathrm{fb}} P_{\mathrm{r}}}+\frac{N_{\mathrm{r}} N_{0}}{N_{0}+N_{\mathrm{pr}} P / N_{\mathrm{r}}}\right)
$$

are the MMSE regularization parameters. Define as $\tilde{\mathbf{H}}_{k}=$ $\left[\tilde{\mathbf{H}}_{k, 1}, \cdots, \tilde{\mathbf{H}}_{k, K}\right]$ the concatenated estimation error matrix. Since writing $\sigma_{\tilde{\mathbf{H}}}^{2}$ exactly yields rather cumbersome expressions and the MMSE solution approaches the ZF (zeroforcing) solution at high SNR, the variance of MMSE error is replaced with that of the ZF solution — as done in [36]—which enables neglecting the constants $\zeta_{1}$ and $\zeta_{2}$. Then, after further simplifications,

$$
\begin{aligned}
\tilde{\mathbf{H}}_{k}= & \sqrt{1+\frac{N_{\mathrm{t}} N_{0}}{N_{\mathrm{pf}} P}}\left[\tilde{\mathbf{H}}_{k}^{\mathrm{f}}+\left(\hat{\mathbf{H}}_{k}^{\mathrm{r} H} \hat{\mathbf{H}}_{k}^{\mathrm{r}}\right)^{-1} \hat{\mathbf{H}}_{k}^{\mathrm{r} H}\right. \\
& \left.\cdot\left(1+\frac{N_{\mathrm{t}} N_{0}}{N_{\mathrm{pf}} P} \tilde{\mathbf{H}}_{k}^{\mathrm{r}} \hat{\mathbf{H}}_{k}^{\mathrm{f}}+\sqrt{\frac{K N_{\mathrm{t}} N_{\mathrm{r}}}{N_{\mathrm{pr}} P_{\mathrm{r}}}} \mathbf{Z} \Theta_{k}^{H}\right)\right]
\end{aligned}
$$

where the entries of $\tilde{\mathbf{H}}_{k}^{\mathrm{f}}$ are

$$
\mathcal{N}_{\mathbb{C}}\left(0, \frac{N_{0}}{N_{0}+N_{\mathrm{pf}} P / N_{\mathrm{t}}}\right),
$$

the entries of $\tilde{\mathbf{H}}_{k}^{r}$ are

$$
\mathcal{N}_{\mathbb{C}}\left(0, \frac{N_{0}}{N_{0}+N_{\mathrm{pr}} P_{\mathrm{r}} / N_{\mathrm{r}}}\right)
$$

and the entries of $\mathbf{Z}$ are uncorrelated $\mathcal{N}_{\mathbb{C}}\left(0, N_{0}\right)$. Utilizing these distributions, the columns of $\tilde{\mathbf{H}}_{k}$ are found to be independent with scaled identity covariance matrices with diagonal entries given by

$$
\begin{aligned}
\sigma_{\tilde{\mathbf{H}}}^{2}= & \frac{N_{\mathrm{t}} N_{0}}{N_{\mathrm{pf}} P} \\
& +\frac{N_{0}}{\left(K N_{\mathrm{t}}-N_{\mathrm{r}}\right) P_{\mathrm{r}}}\left(\frac{N_{\mathrm{r}}^{2}}{N_{\mathrm{pr}}}+\frac{K N_{\mathrm{t}} N_{\mathrm{r}}}{N_{\mathrm{fb}}}\left(1+\frac{N_{\mathrm{r}} N_{0}}{N_{\mathrm{pr}} P_{\mathrm{r}}}\right)\right) .
\end{aligned}
$$

Again, by invoking the high SNR approximation as earlier and replacing the MMSE estimation error with the ZF estimation error, we obtain the final form as in (30).

\section{ACKNOWLEDGMENTS}

The impeccable editorial handling by Prof. Shi Jin, and the excellent feedback provided by the reviewers, are gratefully acknowledged.

\section{REFERENCES}

[1] R. K. Mungara, G. George, and A. Lozano, "Pilot-assisted interference alignment in time-selective fading channels," in Proc. IEEE Global Telecommun. Conf., Dec. 2013, pp. 4082-4087.

[2] S. A. Jafar, Interference Alignment: A New Look at Signal Dimensions in a Communication Network, vol. 7, Foundations and Trends in Communications and Information Theory, 2011.
[3] M. Maddah-Ali and D. Tse, "Completely stale transmitter channel state information is still very useful," IEEE Trans. Inform. Theory, vol. 58, no. 7, pp. 4418-4431, July 2012.

[4] R. Tresch and M. Guillaud, "Cellular interference alignment with imperfect channel knowledge," in Proc. IEEE Int. Conf. Commun., June 2009, pp. 1-5.

[5] B. Nosrat-Makouei, J. G. Andrews, and R. W. Heath Jr., "MIMO interference alignment over correlated channels with imperfect CSI," IEEE Trans. Signal Processing, vol. 59, no. 6, pp. 2783-2794, June 2011.

[6] J. Reitterer and M. Rupp, "Interference alignment in UMTS Long Term Evolution," in Proc. European Sign. Proc. Conf., Barcelona, Spain, Sept. 2011, pp. 1094-1098.

[7] B. Hassibi and B. M. Hochwald, "How much training is needed in multiple-antenna wireless links?," IEEE Trans. Inform. Theory, vol. 49, no. 4, pp. 951-963, Apr. 2003.

[8] B. Xie, Y. Li, H. Minn, and A. Nosratinia, "Interference alignment under training and feedback constraints," in Proc. IEEE Global Telecommun. Conf., Dec. 2011, pp. 1-6.

[9] A. Lozano, J. G. Andrews, and R. W. Heath Jr., "Spectral efficiency limits in pilot-assisted cooperative communications," in Proc. IEEE Int. Symp. Inform. Theory, July 2012, pp. 1132-1136.

[10] J. Thukral and H. Bölcskei, "Interference alignment with limited feedback," in Proc. IEEE Int. Symp. Inform. Theory, June 2009, pp. 1759-1763.

[11] R. T. Krishnamachari and M. K. Varanasi, "Interference alignment under limited feedback for MIMO interference channels," in Proc. IEEE Int. Symp. Inform. Theory, June 2010, pp. 619-623.

[12] M. Rezaee and M. Guillaud, "Limited feedback for interference alignment in the K-user MIMO interference channel," in Proc. IEEE Inform. Theory Workshop, Sept. 2012, pp. 667-671.

[13] O. E. Ayach and R. W. Heath Jr., "Interference alignment with analog channel state feedback," IEEE Trans. Wireless Commun., vol. 11, no. 2, pp. 626-636, Feb. 2012.

[14] N. Jindal, "MIMO broadcast channels with finite-rate feedback," IEEE Trans. Inform. Theory, vol. 52, no. 11, pp. 5045-5060, Nov. 2006.

[15] W. Santipach and M. L. Honig, "Capacity of beamforming with limited training and feedback," in Proc. IEEE Int. Symp. Inform. Theory, Seattle, USA, July 2006.

[16] W. Santipach and M. L. Honig, "Optimization of training and feedback overhead for beamforming over block fading channels," IEEE Trans. Inform. Theory, vol. 56, no. 12, pp. 6103-6115, Dec. 2010.

[17] M. Kobayashi, N. Jindal, and G. Caire, "Traning and feedback optimization for multiuser MIMO downlink," IEEE Trans. Commun. Syst., vol. 59, no. 8, pp. 2228-2240, Aug. 2011.

[18] O. E. Ayach, A. Lozano, and R. W. Heath Jr., "On the overhead of interference alignment: Training, feedback, and cooperation," IEEE Trans. Wireless Commun., vol. 11, no. 11, pp. 4192-4203, Nov. 2012.

[19] Q. Sun, D. C. Cox, H. C. Huang, and A. Lozano, "Estimation of continuous flat-fading MIMO channels," IEEE Trans. Wireless Commun., vol. 1, no. 4, pp. 549-553, oct 2002.

[20] V. Pohl, P. H. Nguyen, V. Jungnickel, and C. V. Helmolt, "Continuous flat-fading MIMO channels: Achievable rate and optimal length of the training and data phases," IEEE Trans. Wireless Commun., vol. 4, no. 4, pp. 1889-1900, July 2005.

[21] N. Jindal and A. Lozano, "A unified treatment of optimum pilot overhead in multipath fading channels," IEEE Trans. Commun., vol. 58, no. 10, pp. 2939-2948, oct 2010.

[22] V. Cadambe and S. A. Jafar, "Interference alignment and the degrees of freedom of the $K$-user interference channel," IEEE Trans. Inform. Theory, vol. 54, no. 8, pp. 3425-3441, Aug. 2008.

[23] K. Gomadam, V. Cadambe, and S. Jafar, "A distributed numerical approach to interference alignment and applications to wireless interference networks," IEEE Trans. Inform. Theory, vol. 57, no. 6, pp. 3309-3322, June 2011.

[24] S. W. Peters and R. W. Heath Jr., "Interference alignment via alternating minimization," in Proc. IEEE Int. Conf. Acoust., Speech, Signal Processing, Apr. 2009, pp. 2445-2448.

[25] L. Ruan, V. K. N. Lau, and M. Z. Win, "The feasibility conditions for interference alignment in MIMO networks," IEEE Trans. Signal Processing, vol. 61, no. 8, pp. 2066-2077, Nov. 2013.

[26] A. Lapidoth and S. Shamai, "Fading channels: How perfect need perfect side information be?," IEEE Trans. Inform. Theory, vol. 48, no. 5, pp. 1118-1134, May 2002.

[27] M. Medard, "The effect upon channel capacity in wireless communications of perfect and imperfect knowledge of the channel," IEEE Trans. on Inform. Theory, vol. 46, no. 3, pp. 933-946, 2000. 
[28] A. Lozano, "Interplay of spectral efficiency, power and Doppler spectrum for reference-signal-assisted wireless communication," IEEE Trans. on Wireless Communications, vol. 7, no. 12, pp. 5020-5029, Dec. 2008.

[29] T. Marzetta, "BLAST training: estimating channel characteristics for high capacity space-time wireless," in Proc. Annual Allerton Conf. Commun., Cont., Computing, Sept. 1999, vol. 37, pp. 958-966.

[30] T. L. Marzetta and B. M. Hochwald, "Fast transfer of channel state information in wireless systems," IEEE Trans. Signal Processing, vol. 54, no. 4, pp. 1268-1278, Apr. 2006.

[31] A. Lozano, A. M. Tulino, and S. Verdú, "High-SNR power offset in multiantenna communication," IEEE Trans. Inform. Theory, vol. 51, no. 12, pp. 4134-4151, Dec. 2005.

[32] A. Ghosh, J. Zhang, J. G. Andrews, and R. Muhamed, Fundamentals of LTE, Prentice-Hall, 2010.

[33] A. Lozano, R. W. Heath Jr., and J. G. Andrews, "Fundamental limits of cooperation," IEEE Trans. Inform. Theory, vol. 59, no. 9, pp. 52135226, Sept. 2013.

[34] R. Mungara, G. George, and A. Lozano, "System-level performance of distributed cooperation," in Proc. Annual Asilomar Conf. Signals, Syst., Comp., Nov. 2012.

[35] D. Aziz, F. Boccardi, and A. Weber, "System-level performance study of interference alignment in cellular systems with base-station coordination," in Proc. IEEE Int. Symp. Pers., Indoor, Mobile Radio Commun., Sydney, Australia, Sept. 2012, pp. 1177-1182.

[36] G. Caire, N. Jindal, M. Kobayashi, and N. Ravindran, "Multiuser MIMO achievable rates with downlink training and channel state feedback," IEEE Trans. Inform. Theory, vol. 56, no. 6, pp. 2845-2866, June 2010.

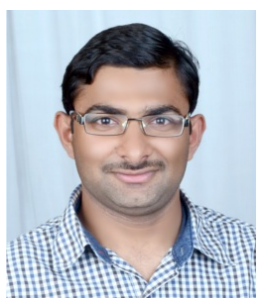

Ratheesh K. Mungara Ratheesh K. Mungara received the B.Tech. degree in Electronics and Communications Engineering from Jawaharlal Nehru Technological University, Hyderabad, India, in 2005, and the M.Tech. degree in Communication Systems from the National Institute of Technology, Tiruchirappalli, India, in 2007.

He is currently working toward his Ph.D. with the Wireless Communications research group, Department of Information and Communication Technologies, UPF (Universitat Pompeu Fabra), Barcelona Spain. He was an engineer at Qualcomm India Private Limited, Hyderabad, from 2007-2010, and a research scientist at the Centre for Wireless Communications (CWC), University of Oulu, Finland, during 2010-2011. His general research interests are modeling and analysis of wireless networks using stochastic geometry tools. His current focus is on interference management techniques and Device-to-Device communication.

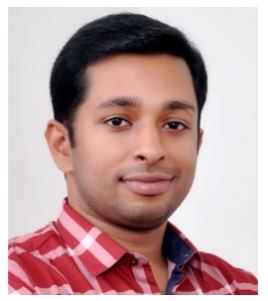

Geordie George Geordie George received his B.Tech. degree in Electronics and Communication Engineering from Kannur University, Kerala, India, in 2004. He completed his M.Tech. degree in Electrical Engineering with specialization in Communication Systems, from the Indian Institute of Technology, Madras, India, in 2007.

Currently, he is a Ph.D. student in the Wireless Communications research group, at the Department of Information and Communication Technologies, UPF (Universitat Pompeu Fabra), Barcelona, Spain. He worked at the Center of Excellence in Wireless Technologies (CEWiT), India, as a research engineer between 2009 and 2011, where he was involved in the standardization activities of IEEE $802.16 \mathrm{~m}$ and 3GPP LTE-Advanced. His research interests are in the areas of communication theory and signal processing, with a focus on the analysis of wireless communication systems. His current interests include the performance analysis of interference management techniques and Device-to-Device (D2D) integrated cellular networks.

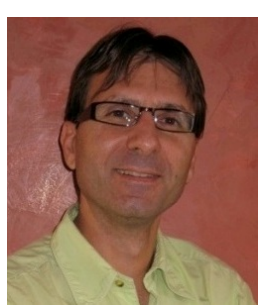

Angel Lozano Angel Lozano is a Professor of Information and Communication Technologies at UPF (Universitat Pompeu Fabra) in Barcelona, Spain. He received the MSc. and Ph.D. degrees in Electrical Engineering from Stanford University in 1994 and 1998, respectively. In 1999 he joined Bell Labs (Lucent Technologies, now Alcatel-Lucent) in Holmdel, USA, where he was a member of the Wireless Communications Research Department until 2008. Between 2005 and 2008 he was also Adj. Associate Professor of Electrical Engineering at Columbia

University.

Prof. Lozano is a Fellow of the IEEE since 2014. He is an associate editor for the IEEE Transactions on Information Theory (since 2011), a former editor for the IEEE Transactions on Communications (1999-2009) and the Journal of Communications \& Networks (2010-2012), has guest-edited various journal special issues, and is actively involved in committees and conference organization tasks for the IEEE. In particular, he is the Chair of the IEEE Communication Theory Technical Committee (2013-2014) and was elected to the Board of Governors of the IEEE Communications Society (2012-2014). $\mathrm{He}$ has further participated in standardization activities for 3GPP, 3GPP2, IEEE 802.20 and the IETF.

Prof. Lozano has published extensively, holds 15 patents, and has contributed to several books. His papers have received two awards: the best paper at the 2006 IEEE Intl Symposium on Spread Spectrum Techniques \& Applications, and the Stephen O. Rice prize to the best paper published in the IEEE Transactions on Communications in 2008. He also received the Bell Labs Presidents Gold Award in 2002 and the ICREA Academia Award from the Catalan Government in 2011. 